\title{
ÉCONOMIE DE LA TRANSMISSION PÉGUY APRÈS WALRAS
}

\author{
Michele Bee ${ }^{1}$ \\ Centre Walras Pareto, Université de Lausanne
}

La mort de Charles Péguy pendant la Première Guerre mondiale mit fin à son œuvre ainsi qu'à l'entreprise des Cahiers de la Quinzaine. Cet espace de liberté intellectuelle $n^{\prime} a$ pas été le fruit du sacrifice d'un esprit étranger aux questions économiques mais le résultat d'une gestion profondément attentive à la réalité économique. Une attention que Péguy a manifestée depuis son tout premier article, ouvrant ainsi en tant que jeune normalien un dialogue avec l'un des initiateurs reconnus de l'économie mathématique, Léon Walras. La correspondance épistolaire entre ces deux penseurs montre l'intérêt que chacun porte aux idées de l'autre ainsi que la distance qui les sépare. Si Péguy n'est pas en soi opposé à l'application des mathématiques en économie, il critique la façon dont elles affectent la théorie de Walras en ne lui permettant pas de prendre en compte l'hétérogénéité des phénomènes économiques. Ce qui signifie, pour Péguy, de ne pas pouvoir considérer dès le début que tout acteur économique subit ou transmet inévitablement des pressions sociales. Or, cette prise en compte de l'économie de la transmission permettrait en retour d'évaluer une organisation non pas tant sur la base de sa forme juridique que sur sa capacité à absorber de telles pressions. De ce point de vue, les Cahiers de la Quinzaine n'apparaissent plus comme une entreprise éditoriale plombée par un équilibre financier précaire, qui a consumé la vie de son fondateur. Ils illustrent plutôt ce que Péguy n'a jamais cessé d'indiquer à ses lecteurs : la tentative de proposer une alternative à l'entreprise typiquement capitaliste ainsi qu'à toute forme d'étatisme. Une alternative à laquelle il aurait pu continuer à travailler pratiquement et théoriquement s'il était revenu du champ de bataille.

Mots clés : Péguy, Walras, Bergson, pressions sociales, gestion ouverte.

1. Je tiens à remercier Massimo Amato, Pascal Bridel, Raphaël Fèvre, Philip Gerard, Antoine Parent, Alexandre Schild et Vincent Touzé pour leurs précieuses suggestions, ainsi que le Fonds Walras de la Bibliothèque de I'Université de Lausanne pour la mise à disposition des lettres de Charles Péguy et de Léon Walras. 
-e 4 août 1914, Charles Péguy part en guerre pour défendre les idéaux qui ont toujours été les siens. II part «pour le désarmement général, pour la dernière des guerres ${ }^{2}$, contre le «militarisme » et $\mathrm{I}^{\prime}$ « impérialisme » incarnés alors par le pangermanisme ${ }^{3}$. À la veille de la contre-offensive française de la Marne, Péguy est tué sur le champ de bataille à l'âge de 41 ans. À sa mort, la revue libertaire allemande Die Aktion lui dédiera sa couverture, mettant en pleine page son portrait dessiné par Egon Schiele ${ }^{4}$. Si Péguy a pu être vu pendant la guerre comme une figure exemplaire par des intellectuels allemands, c'est par la force et la clarté de son engagement pour la liberté de tous les peuples, l'élevant au rang de conscience européenne. Son œuvre continuera d'exercer un profond magistère à travers une approche tout à la fois littéraire, poétique et philosophique, aussi bien sociale qu'économique. Ce dernier point est peut-être celui qui a reçu le moins d'attention dans les études dédiées à son œuvre. Pourtant, la mort de Péguy mettra effectivement un terme à une action et à une œuvre profondément économique qui trouve son couronnement dans l'expérience des Cahiers de la Quinzaine.

Dans une lettre du 19 février 1897 (voir document 1 en annexe), le jeune étudiant Charles Péguy confie à l'économiste Léon Walras : «Je ne sais pas si les nécessités de l'action me laisseront, comme je le voudrais bien, le temps et les moyens de travailler à l'économie mathématique » (OPC I, p. 1546) ${ }^{5}$. Il peut sembler surprenant de voir Péguy parler à Walras de son intérêt pour l'économie mathématique. En fait, les deux hommes sont entrés en contact parce que la Revue socialiste sollicita Péguy pour un compte rendu des Études d'économie sociale (Théorie de la répartition de la richesse sociale) de Walras $(1896)^{6}$.

Le jeune normalien a été ainsi le premier en France à faire un compte rendu de ce travail, publié le 19 février 1897 avec le titre « Un

2. D'après ce que Péguy aurait dit à Geneviève Favre au moment de son départ, ainsi que l'a reporté Daniel Halévy (1941, p. 380).

3. Si dans Notre Jeunesse (1910) Péguy se revendique d'un socialisme «exactement internationaliste", dans L'Argent suite (1913, p. 945) il déplore un internationalisme devenu un pangermanisme, c'est-à-dire « un total asservissement à la politique allemande, au capitalisme allemand, à l'impérialisme allemand, au militarisme allemand, au colonialisme allemand ».

4. Voir Die Aktion. Wochenschrift für Politik, Literatur, Kunst, $n^{\circ}$ 42-43, Berlin-Wilmerdorf (1914).

5. Les lettres de Walras - plus exactement les brouillons de ses lettres - et celles de Péguy sont conservées à la Bibliothèque cantonale et universitaire de Lausanne. Cette correspondance a été publiée pour la première fois dans Jaffé (1965). Elle est citée ici à partir des extraits publiés dans Charles Péguy, đuuvres en prose complètes (OPC), vol. I, édité par Robert Burac, Paris, Gallimard (1987, pp. 1545-1548). 
économiste socialiste, M. Léon Walras », et surtout il a été parmi les rares à l'époque à en comprendre l'importance (voir Dockès, 1996 ; Bridel, 1996). Walras tente de l'encourager à poursuivre l'étude de l'économie mathématique mais Péguy ne semble pas déterminé à le faire, ce qui pourrait amener à penser qu'il s'est agi d'une « séduction » momentanée vis-à-vis de laquelle il se serait rapidement affranchi (Thibaud 1975) ${ }^{7}$. En fait, toute de suite après le compte rendu, la vie de Péguy a l'air de prendre un tout autre chemin (Delaporte, 1967).

Le présent article propose une réinterprétation de la réception critique de Walras par Péguy en mettant en lumière l'importance du cadre bergsonien au sein duquel ce dernier raisonne. À partir de cette nouvelle lecture, l'article entend montrer qu'à partir de sa lecture initiale de Walras, Péguy n'a pas abandonné mais bien poursuivi son projet d'une autre théorie économique, dont les principes sont issus de sa propre expérience commerciale en tant que gérant des Cahiers de la Quinzaine.

Si la discussion entre Walras et Péguy a déjà fait l'objet d'une certaine attention, un élément crucial semble cependant $n$ 'avoir pas été pris suffisamment en considération : l'intérêt contextuel et probablement antérieur de Péguy pour Bergson. On sait que Péguy propose dans ses écrits plus tardifs une lecture tout à fait personnelle de la pensée de Bergson, notamment dans le but de l'étendre du plan intérieur de la conscience au plan historique et social ${ }^{8}$. Mais ce que l'on sait moins, $c^{\prime}$ est que Péguy a commencé à travailler dans cette perspective dès ce compte rendu sur les Études d'économie sociale ${ }^{9}$. C'est probablement son approche bergsonienne qui l'a attiré vers l'œuvre de Walras, lui faisant accepter la proposition d'en faire le compte rendu. Si Bergson s'intéresse à la mécanique mathématique pour indiquer le

6. Le directeur de la Revue socialiste, Georges Renard, est un collègue de Walras à l'Université de Lausanne. Il est aussi l'oncle d'un des camarades de Péguy à l'École normale supérieure, Georges Weulersse. Si Walras essaie de faire connaître son travail en France à travers son collègue, Weulersse semble persuader son oncle de l'importance de la figure de Péguy chez les jeunes socialistes de l'École normale (voir Smith 1973, p. 258). C'est ainsi que Péguy reçoit la proposition de Renard de commenter les Études de Walras.

7. Sur le rôle qu'a pu avoir le cours de Charles Andler à l'École normale dans cet intérêt de Péguy pour l'économie mathématique, voir Leroy (1973).

8. Cela est particulièrement visible dans le dernier texte publié par Péguy, Note sur M. Bergson et la philosophie bergsonienne (1914) ainsi que dans les textes posthumes Clio, dialogue de l'histoire et de l'âme païenne (1912-3) et Note conjointe sur M. Descartes et la philosophie cartésienne (1914), mais aussi déjà dans ses premiers textes sur les Cahiers (à ce propos, voir entre autres Riquier, 2014 ; Creyghton, 2011 ; Bédarida, 2002 ; Gerbod, 2002). On sait que Bergson lui-même dira que Péguy est allé jusqu'où lui-même n'avait pas encore osé aller. 
temps intérieur qui échappe au temps indéfini et homogène de cette science, Péguy s'intéresse à l'économie mathématique pour mettre en évidence le temps historique qui lui échappe dans la recherche de la solution à la question sociale. Comme Bergson, Péguy porte une grande attention à la méthode mathématique et cela le distingue certainement de la plupart des autres commentateurs des travaux de Walras de l'époque, qui ont tendance à rejeter a priori l'application de cette méthode aux sciences humaines (voir Dockès, 1996).

Pour Péguy, en revanche, le problème n'est pas de compter mais de savoir comment et quoi compter, car quelque chose échappe toujours au calcul et toutes les façons de compter ne sont pas les mêmes. Suivre la critique que porte Bergson à l'encontre des symboles mathématiques ou linguistiques ne signifie pas pour Péguy s'empêcher de compter ou de parler. II s'agit plutôt de savoir comment compter et parler de façon à rester le plus fidèle possible à la réalité (voir aussi Worms, 2000) ${ }^{10}$. Mettre en évidence l'adoption d'une perspective bergsonienne par Péguy dans le compte rendu qu'il fait des Études permet donc de mieux cerner sa critique de l'économie walrassienne ainsi que l'évolution de sa propre pensée économique. Cela signifie aussi que le bergsonisme de Péguy ne s'est pas construit comme réponse à sa déception pour la pensée socialiste, après qu'il eut écrit « dans la ligne de Jaurès, de Georges Renard et de Walras » (Robinet, 1968 , p. 19) ${ }^{11}$. C'est plutôt la pensée de Bergson qui, tout en donnant corps à l'intérêt de Péguy pour l'économie mathématique, a marqué de son pli I'adoption de sa perspective socialiste singulière ${ }^{12}$.

9. Le premier contact certain de Péguy avec Bergson eut lieu pendant les cours de ce dernier à I'École normale en février 1898, exactement un an après la publication du compte rendu sur Walras. On sait cependant qu'au moment où il rédige ce texte, Péguy non seulement connaît déjà l'œuvre de Bergson, mais l'apprécie à tel point que, dans cette même année 1896, il offre Matière et Mémoire à Charlotte Badouin en cadeau de fiançailles. Péguy lui-même a affirmé dans les Cahiers du 4 juillet 1900 que lorsqu'il a commencé à suivre les cours de Bergson à l'École normale, il avait déjà lu Essai sur les données immédiates de la conscience (1889) et Matière et Mémoire (1896).

10. Le style répétitif avec lequel Péguy va utiliser la langue tout en essayant de rester le plus fidèle possible à la réalité est bien connu (Latour, 2014 ; Saatdjian, 2001). Dans cet article, nous nous concentrons plutôt sur la manière dont Péguy cherche à compter, tout en essayant de ne pas rester captif de la méthode quantitative.

11. Comme souligné par Saatdjian (2011), le parcours de Péguy observe plutôt une forme de continuité travaillée par un mouvement d'approfondissement.

12. En 1910, Péguy écrit comme dédicace à Bergson sur un exemplaire de sa Jeanne d'Arc (1897) qu'à l'époque de l'écriture de ce texte ils ne se connaissaient pas encore, mais qu'il était en fait déjà un ses élèves, et que cette amitié a muri pour 15 ans, c'est-à-dire depuis 1895, date à laquelle il aurait lu l'Essai sur les données immédiates de la conscience (1889). Toutefois, le compte rendu de Walras par Péguy ne devrait pas être lu comme un texte scolaire sous l'influence de Bergson, la pensée de Péguy étant dès le début déjà celle d'un philosophe en dialogue avec un autre philosophe (sur Péguy philosophe, voir Riquier, 2017 ; Fédier, 2014). 
En interprétant la pensée de Bergson, Péguy tente de rendre compte de quelque chose que Walras est obligé de négliger dès ses premiers pas, c'est-à-dire l'hétérogénéité de la réalité. Ce dernier commence son étude en supposant que la concurrence est parfaite et que les individus sont comme des éléments homogènes se mouvant dans une temporalité indéfinie. Mais, chaque fois que la concurrence n'est pas parfaite, comme c'est souvent le cas, note Péguy, I'hétérogénéité réapparaît. Une telle hétérogénéité implique que le temps économique ne peut pas être représenté sur une ligne, comme le voudrait Walras de façon cartésienne. Si ce dernier émet l'hypothèse d'un idéal théorique parfait dans une temporalité indéfinie, Péguy pense qu'il est plus approprié d'expérimenter une action qui, aussi imparfaite soit-elle, vise à résoudre la question sociale en tenant compte du temps historique. Si Walras part d'individus théoriquement libres de toute contrainte, Péguy entend d'abord étudier comment les contraintes sont transmises dans la réalité afin de comprendre comment s'en libérer au mieux.

D'où le choix de l'action. Au sens bergsonien, après tout, seule l'action permet de ne pas rester prisonnier de symboles qui ne saisissent pas la réalité dans sa globalité (voir Guerlac, 2006). II est évident que l'action qui intéresse Péguy est celle visant à résoudre la question sociale. Cette question pour lui n'est pas donnée par le problème de savoir comment répartir de la manière la plus juste la richesse produite dans la société idéale et future mais de comprendre comment partager la perte aujourd'hui. Le véritable enjeu pour Péguy n'est pas le problème walrassien de savoir qui va boire le Château-Lafite dans la société idéale, mais celui de résoudre aujourd'hui le problème de la misère et de l'éducation. C'est probablement ce que Péguy entend dire à Walras quand il lui parle des nécessités de l'action. Peu de temps après, il quittera l'École normale supérieure et créera la librairie socialiste Georges-Bellais, prélude à I'aventure des Cahiers de la Quinzaine. II ne s'agit donc pas d'un changement de cap dans la vie de Péguy mais d'un choix cohérent avec ce qu'il a annoncé à Walras dans leur correspondance et avec ce qu'il a publié quelques jours plus tôt dans son compte rendu. En fait, c'est précisément à partir de l'expérience pratique des Cahiers que Péguy va puiser les éléments pour mettre en place une économie différente de celle suggérée par Walras.

Au cours de trois conférences données en 1904 à l'École des hautes études sociales - dans lesquelles il précise explicitement sa démarche personnelle bergsonienne (voir Worms, 2000) - Péguy propose un 
véritable retournement de méthode informé par ce qu'il a déjà appris de l'expérience pratique de son entreprise éditoriale ${ }^{13}$. Tout comme pour Bergson les états de conscience se pénètrent les uns les autres dans I'hétérogénéité de la conscience, pour Péguy les personnes sociales se pénètrent les unes les autres dans la communication sociale. Selon l'approche de Bergson, il faut partir du mouvement et non des points d'arrêt. C'est pourquoi Péguy tente de mettre en place une économie qui n'a pas le péché originel de l'économie walrassienne et qui part de la communication sociale pour ensuite atteindre les personnes sociales, au lieu de partir de ces dernières en essayant de les réassembler vainement plus tard dans une temporalité abstraite. De ce point de vue, la communication sociale doit être le point de départ et non le point final des études sociales. Pour tenter de résoudre la question sociale autant que possible, il faut donc étudier la transmission de la pression économique et donc essayer de comprendre comment l'absorber (voir aussi Amato, 2009 ; Riquier, 2017). À cet effet, Péguy distingue les autorités de commandement des autorités de compétence et énonce une forme de maxime où la quasi-disparition des premières permettrait aux autorités de compétence de s'équilibrer dans l'échange social. Absorber la pression sociale ne signifie donc pas réduire à zéro toute la communication sociale mais la rendre aussi libre que possible. De sorte que la valeur sociale d'une entreprise ne doit donc pas être jugée en fonction de sa forme juridique - qu'elle soit capitaliste ou collectiviste - mais en fonction de sa force de libération.

C'est ainsi que Péguy nous invite à juger son entreprise. On se souvient à juste titre des Cahiers tant pour la liberté qui y régnait que pour l'effort héroïque avec lequel ils ont été conduits (Vitry, 2015). Mais cet « héroïsme » invite souvent à lire cette expérience comme peu instructive d'un point de vue économique : la poursuite par Péguy d'un idéal de libre expression absolue a non seulement ruiné sa santé mais maintenu à flots une entreprise non viable (Leroy, 2014 ; 1975). Les difficultés financières des Cahiers sont incontestables, comme le souligne Péguy lui-même à plusieurs reprises. Mais en même temps, s'il en parle, c'est pour indiquer à l'aune de quels critères cette entreprise doit être jugée. Si l'on considère les pressions reçues et les pressions transmises, les Cahiers apparaissent alors comme un précédent, comme une tentative d'entreprise socialiste viable. Les inventions

13. Ces conférences ont été publiées à titre posthume pour la première fois dans Viard (1969, pp. 110-150). 
administratives adoptées pour absorber la pression exercée sur les Cahiers sont un exemple qui permet d'illustrer concrètement les réflexions économiques que Péguy a su tirer de cette expérience. D'ailleurs, comme il l'a dit lui-même : «Tous les articles que l'on peut mettre dans une revue, notamment les articles de théorie économique, ne vaudront jamais une bonne histoire économique de la revue ellemême » (Péguy, 1906, p. 407).

\section{Vers l'équilibre économique général de Walras : une critique bergsonienne}

\subsection{Abstraire l'homogénéité de l'hétérogénéité}

Pour faire son compte rendu des Études d'économie sociale de Walras, Péguy étudie et discute également ses Éléments d'économie politique pure (1874), dans lesquels l'économiste a jeté les bases de sa méthode scientifique et mathématique ${ }^{14}$. Pour Péguy, il est clair que cette méthode ne doit pas du tout être sous-estimée, contrairement à ce qui pensent la plupart des économistes de l'époque (voir Dockès 1996). Péguy défend Walras contre les critiques faciles, au point de soutenir que ce qui fait la force de son argumentaire repose moins sur ses principes économiques que sur sa méthode ${ }^{15}$ :

La méthode mathématique est si peu superflue en pareille matière que c'est elle qui a donné à $M$. Walras, pour la bataille des idées économiques, cet avantage indéniable qu'il croit devoir à ses principes. (Péguy, 1897a, pp. 11-12)

Trois jours après la publication du compte rendu, Walras écrit à Péguy pour le féliciter: " toute la partie d'analyse est excellente de conscience, de pénétration et d'exactitude » (Walras, OPC I, p. 1545). Cette appréciation est d'une certaine importance si l'on tient compte du fait que Péguy analyse la pensée de Walras à la lumière d'une approche bergsonienne implicite.

14. Péguy emprunte les Éléments à la bibliothèque de l'École normale le 31 décembre 1896 et le rend le 24 mars 1897 (voir Dockès, 1990). Sur le rapport entre les Éléments et les Études dans la pensée de Walras, voir Potier (1994).

15. Péguy rejette deux autres critiques qui sont faites à Walras sur l'utilisation de la méthode mathématique en économie. Certains pensent que la réalité est trop complexe pour être simplifiée avec cette méthode; cependant, ils ne tiennent pas compte du fait que même les premières propositions de la géométrie sont très simples, ce qui n'interdit pas d'autres complications. D'autres encore craignent qu'une telle méthode ne conduise à se complaire dans une théorie qui ne fonctionne que dans l'abstraction; mais ils ne tiennent pas compte du fait que, pour Walras, la théorie sert à montrer non seulement l'idéal à atteindre, mais aussi le chemin pour y parvenir (voir Péguy, 1897a, pp. 12-3). 
Bergson s'intéresse à la mécanique et à la physique pour montrer que quelque chose échappe à ces sciences à cause de leur méthode : en représentant le passage du temps comme une succession de points homogènes et distincts sur une ligne, elles perdent la possibilité de considérer la durée, c'est-à-dire le temps qualitatif qui reste nécessairement « en dehors de l'équation » (Bergson, 1899, p. 89) ${ }^{16}$. Lorsque ces sciences comptent le temps, elles ne considèrent que les extrémités de ces intervalles identiques et distincts, les points de repos, statiques, et non le mouvement du temps, sa durée d'un point à un autre. Ce qui implique que ces extrémités sont considérées séparément les unes des autres. Dans une mélodie, au contraire, les éléments passés sont indissolublement liés aux éléments suivants : ils s'interpénètrent les uns les autres, constituant une continuité très proche de celle propre aux états de conscience hétérogènes. En suivant cette approche, Péguy s'adresse à l'économie mathématique de Walras pour montrer ce qui lui échappe : en représentant les individus comme des éléments homogènes et distincts et non comme des personnes sociales qui se pénètrent les unes les autres, cette science perd la possibilité de considérer le temps historique d'une communauté humaine, sa réalité continue et hétérogène. Aussi implicite ce programme soit-il, Péguy en indique les fondements dès le début de son compte rendu :

En étudiant les phénomènes économiques généraux relatifs soit aux États soit aux individus selon les méthodes mathématiques, M. Léon Walras a vraiment instauré une science. II nous en a donné les éléments dans ses Éléments d'économie politique pure et, comme nous avions la mécanique mathématique, la physique mathématique, aussi exactement et dans le même sens nous avons à présent l'économie mathématique. (Péguy, 1897a, p. 3, italiques ajoutés)

Tout de suite après, Péguy explique avec une terminologie bergsonienne ce que signifie avoir une économie mathématique dans le même sens qu'on avait auparavant la mécanique mathématique et la physique mathématique. Dans l'économie de Walras, il est possible de représenter sur des courbes les variations des quantités considérées et de trouver la solution aux problèmes qui sont posés par un système d'équations en équilibre. C'est ainsi que Walras peut atteindre

16. Selon Bergson, même la mécanique mathématique qui prend en compte les intervalles de temps les plus infinitésimaux ne considère que les extrémités : si l'univers allait deux fois plus vite, rien ne changerait dans les formules et les équations de la science astronomique; pourtant, notre conscience serait perturbée. Il y a donc une distinction entre la durée du temps réel perçu intérieurement et le temps mathématique appliqué à la réalité qui donne lieu à un temps homogène (voir Riquier, 2009 ; Worms, 1994). 
l'équilibre économique général. Cependant, pour que cette nouvelle science soit possible, son objet ne doit pas être étranger à ses méthodes : si les phénomènes économiques «nous sont donnés, en un sens, très hétérogènes » alors il faut « de ces phénomènes hétérogènes, abstraire les éléments homogènes à qui seuls conviennent les méthodes mathématiques»(Péguy, 1897a, p. 3, italiques ajoutés). Pour l'économie mathématique, cela signifie qu'à partir d'une concurrence réelle "hétérogène », composée de «contraintes » et exercée par des individus «inégaux » entre eux, Walras abstrait une concurrence homogène entre des individus égaux (ibid., p. 4). Partant donc d'individus libres de contraintes réciproques, c'est-à-dire indépendants les uns des autres, Walras les met en communication par le biais du troc. Il va confronter le troc de Stanley Jevons à celui de HermannHenri Gossen. Selon Jevons, chacun des troqueurs prend sa décision de façon à se procurer la plus grande somme possible de satisfaction de ses besoins. Selon Gossen, par contre, pour arriver au point de maximum absolu de satisfaction des besoins des deux troqueurs, il faut considérer les deux individus pris ensemble, abstraction faite du droit de propriété ${ }^{17}$. Comme seul le troc de Jevons respecte à la fois la propriété individuelle et la libre concurrence, Péguy montre que Walras le choisit pour une question de méthode générale (trouver une solution respectueuse de la propriété et de la concurrence) mais aussi en raison de la méthode mathématique adoptée : ce n'est qu'à la suite de ce type de troc, et non de celui de Gossen, que les individus se retrouvent à nouveau dans une position d'indépendance les uns vis-àvis des autres. En tant qu' « unités possédantes », le troc de Gossen les altère au sens où il ne les laisse pas identiques à eux-mêmes; le choix de cette modalité du troc réintroduirait l'hétérogène au commencement de la science nouvelle (Péguy, 1897a, pp. 5-6). En tant qu' « unités concurrentes », le troc de Gossen suppose qu'ils ne sont pas libres; mais il suffit que la concurrence ne soit pas exactement libre pour que l'hétérogène, par-là, se trouve encore une fois réintroduit au commencement de la science (ibid.) ${ }^{18}$.

17. Dans le troc de Jevons, qui est défini par Walras $d^{\prime}$ « individualiste », la condition du maximum est que, une fois le troc effectué, «le rapport des intensités des derniers besoins satisfaits soit, chez chaque troqueur, égal au prix d'une marchandise en l'autre " (Walras, 1896, p. 179). Dans le troc de Gossen, les deux marchandises sont réparties entre les deux troqueurs de telle sorte que «l'intensité du dernier besoin satisfait de chaque marchandise soit la même pour l'un et l'autre » (ibid., p. 181). Dans ce cas, nous sommes en face d'un troc qui, selon Walras, est basé sur le principe de la «fraternité ». 


\subsection{La solution arrive toujours trop tard}

En adoptant le troc de Jevons, l'échange en libre concurrence de Walras ne produit aucune injustice : à la fin de chaque échange, la composition des biens de chacun est modifiée de manière à augmenter I'utilité individuelle sans changer la valeur initiale de ces biens (voir Bridel et Huck, 2002). Cela signifie toutefois que si les conditions de départ ne sont pas justes, elles ne peuvent pas devenir plus justes grâce au système de marché en libre concurrence. Puisque Walras ne souhaite pas s'écarter de la libre concurrence pour les raisons de méthode exposées ci-dessus, il doit alors trouver une autre solution à la question sociale. Pour Walras, une condition initiale est injuste lorsque les individus ne jouissent pas du fruit de leurs facultés individuelles et la communauté dans son ensemble de ses facultés collectives. De sorte qu'il ne devrait pas y avoir d'imposition fiscale, car cela empêche les particuliers d'obtenir le plein fruit de leur travail. Mais l'imposition fiscale est due au fait que l'État ne peut pas payer les services publics avec la rente foncière, qui est le fruit des facultés collectives, parce que les terres ont été injustement privatisées.

Avec sa « théorie mathématique du prix des terres et de leur rachat par l'État », Walras envisage une nationalisation de la terre non pas par une expropriation violente mais par l'échange en libre concurrence. Le stratagème repose sur le fait que les propriétaires terriens, au moment du rachat de leurs terres par l'État à un prix courant, ne peuvent pas revendiquer la rente supplémentaire qui sera générée par le passage d'époque d'une société agricole à une société industrielle et commerciale : quand ils les ont achetées ils n'ont pas pu anticiper dans le prix de leur achat ce montant de rente supplémentaire (voir Dockès, 1996). Après un nombre $m$ d'années, cette rente supplémentaire devrait permettre à l'État d'amortir la dette contractée pour le rachat des terres ainsi que d'assurer les services publics sans avoir recours à l'impôt, donc en laissant fonctionner le marché selon le principe de la libre concurrence. C'est à partir de ce moment que selon Walras la question sociale sera résolue : «Après $m$ années - explique Péguy - commencera le règne souverain du droit social et son avènement n'aura pas altéré du tout l'être économique ; après $m$ années aura lieu, pour ainsi dire,

18. Dans la perspective de Gossen les individus sont toujours liés et dépendants les uns des autres ; c'est pourquoi il n'est pas possible d'établir une variable indépendante. Autrement dit : il n'est pas possible d'établir a priori les paramètres d'une fonction de superposition des fonctions d'utilité, c'està-dire une loi mathématique générale des échanges. 
I'intersection du droit désormais entier et de l'être toujours intact. Et telle est la solution que $\mathrm{M}$. Walras nous propose de la question sociale » (Péguy, $1897 a$, p. 11). ${ }^{19}$ Après $m$ années, on arriverait à une situation dans laquelle on aurait obtenu une juste répartition de la richesse sans avoir exproprié aucun propriétaire.

Cependant, dit Péguy, ce point $m$ arrive toujours trop tard. "Préparer de la justice définitive et lointaine - écrit-il - avec de l'injustice intermédiaire et prochaine, cela n'est pas juste » (ibid., p. 17). Le problème ne réside pas, cependant, dans le fait que la solution à la question sociale de Walras arrive trop tard, en ce sens qu'elle devrait arriver plus tôt sur la même ligne de temps, mais dans le fait que ce dernier pense le temps comme s'il s'agissait d'une ligne indéfinie et homogène, composée de points distincts et indépendants les uns par rapport aux autres. D'après Péguy, ce procédé serait somme toute valable tant que les années sont considérées «comme des années arithmétiques, découpées dans un temps mathématique infini et indifférent à la position du présent »; dans ce cas-là, il suffit « de figurer jusqu'à l'intersection par un trait plein l'être économique et par un trait ponctué le droit social » (ibid., p. 16). Mais, selon Péguy, « ces années ne peuvent pas se calculer ni se figurer comme des années arithmétiques", car cela rend impossible la prise en compte de la situation présente (ibid.) ${ }^{20}$. La solution de Walras est inévitablement toujours en retard car elle ne tient pas compte du fait qu'étant injuste au départ, tout ce qui suit est inévitablement conditionné par cette injustice séminale. S'il est vrai, comme le dit Walras, que l'État est le propriétaire légitime du sol, alors « il suffit que ce propriétaire individuel détienne ce sol pour qu'il en dépossède incessamment l'État ", qui est donc obligé d'utiliser la rente supplémentaire pour compenser les pertes injustement encourues, c'est-à-dire la dette contractée pour le rachat des terres (ibid.).

En substance, quelque chose échappe nécessairement aux études économiques si elles considèrent les individus exclusivement comme des éléments homogènes et distincts, comme le sont les points sur une «ligne mathématique ordinaire » (ibid., 15). Ce qui échappe, c'est la

19. Sur le réalisme téléologique de Walras, voir Baranzini et Fèvre (2019).

20. «Des années économiques hétérogènes - écrit Péguy - le mathématicien peut bien abstraire des années homogènes : il ne saurait, d'années constituées en série définie et posée à l'égard d'un présent donné, abstraire une série indéfinie d'années, une série indifférente à la position du présent » (1897a, p. 16). 
temporalité historique de la communauté à laquelle ils appartiennent, temporalité qui comprend dans ce cas une répartition injuste de la terre ; ce qui implique, selon Péguy, que la solution mathématique de Walras à la question sociale est en retard dès le départ, en retard par construction. Pour Péguy, cependant, il faut considérer qu'il suffit que l'individu soit donné pour qu'inséparablement soit donné aussi la communauté dont relève cet individu. Ce qui veut dire que si l'on entend représenter mathématiquement un individu, il faut à chaque fois le rapporter au nombre total des individus auxquels il est d'avance lié : «si, ayant en économie à représenter neuf hommes, on les représente seulement par le chiffre 9, on est déjà inexact, parce que l'expression paraît complète et qu'elle ne l'est pas» (ibid., p. 15). Supposant que le nombre total des hommes au moment considéré soit égal à quinze cents millions, pour être exact - écrit Péguy - il faudrait représenter ces neuf hommes par une fraction avec 9 au numérateur et quinze cents millions au dénominateur ${ }^{21}$.

\subsection{Compter autrement}

Après avoir pris connaissance du compte rendu de Péguy, Walras lui demande de lui envoyer un manuscrit dans lequel il aborde plus longuement la question de l'économie mathématique, incluant les œuvres de Cournot, Gossen et Jevons. Péguy lui envoie ce texte en disant que c'est «une enquête philosophique sur l'économie mathématique et non point une contribution à cette science " (Péguy, OPC I, p. 1546) ${ }^{22}$. Walras lui écrit à nouveau le 31 mars 1897 (voir document 2 en annexe). II se dit très intéressé par ses réflexions, et après avoir assuré Péguy qu'il a lu son manuscrit trois fois de suite, il dit vouloir discuter expressément d'un point qui lui semble sensible: " c'est celui qui figurait dans votre article de la Revue socialiste et que j'ai retrouvé dans votre travail et qui consiste à dire que l'opération mathématique par laquelle on considère $n$ hommes ou $n$ francs sur $T_{h}$ hommes ou $T_{f}$ francs formerait un symbole incomplet et inexact. Je ne

21. Si on tient compte de ce que dit Péguy, en considérant tous les individus pour faire la somme, le résultat est l'entier: $n_{1} / T_{\mathrm{h}}+n_{2} / T_{\mathrm{h}}+n_{3} / T_{\mathrm{h}}+\ldots+n_{T h} / T_{h}=1$ au lieu du total $: n_{1}+n_{2}+n_{3}+\ldots$ $n_{T h}=T_{\mathrm{h}}$.

22. Malgré l'intérêt manifeste de Walras, ce dernier indique également que Péguy se tient « exclusivement sur le terrain philosophique » où il ne se sent pas à son aise. Et il ajoute : « Je suis économiste surtout. [...] Vous voyez d'ici ma position: elle est celle d'un astronome ou d'un physicien devant lequel des philosophes discuteraient abstraitement et a priori, soit pour l'affirmer, soit pour la nier, la légitimité et l'utilité de l'emploi des mathématiques en astronomie et en physique » (Walras, OPC I, p. 1547). 
crois pas avoir, pour ma part, procédé de cette façon » (Walras, OPC I, p. 1547). Walras répond donc ainsi à la critique de Péguy :

La mathématique nous permet de faire entrer en ligne de compte, avec trois points, tous les hommes et tous les francs. En économie politique pure, quand je dis : soient les marchandises (A), (B), (C), (D)..., soient les échangistes (1), (2), (3) ..., je considère toutes les marchandises et tous les échangistes sans exception.

(Walras, OPC I, p. 1547)

Nous ne savons pas ce que Péguy a écrit dans son manuscrit, car il n'a pas été retrouvé. Toutefois, comme le souligne Walras, le point qui I'intéresse se retrouve également dans le compte rendu. Dans celui-ci, toutefois, Péguy ne dit pas que ne considérer que 9 ou $n$ hommes sur $T_{h}$ hommes formerait un symbole incomplet et inexact et que Walras aurait opéré de cette façon. Pour Péguy, il ne s'agit pas d'être incomplet et inexact en ne considérant pas tous les échangistes et toutes les marchandises, mais qu'en prenant en compte tous les échangistes et toutes les marchandises, même sans exception, on ne considère pas que chacun de ces éléments fait partie d'une "solidarité circulaire » (Péguy, 1897a, p. 15). Ce discours en effet peut sembler difficile à suivre (voir Thibaud, 1985, p. 140), surtout si l'on ne tient pas compte du fait que Péguy se réfère probablement ici aussi à l'approche bergsonienne ${ }^{23}$. Pour Bergson, les états intérieurs de la conscience se succèdent sans distinction comme les notes d'une mélodie, «comme une pénétration mutuelle, une solidarité, une organisation intime d'éléments, dont chacun, représentatif du tout, ne s'en distingue et ne s'en isole que pour une pensée capable d'abstraire » (Bergson, 1899). De la même façon pour Péguy, les personnes ne sont pas liées entre elles «comme les unités du nombre mathématique ordinaire ou comme les points de la ligne mathématique ordinaire, mais sont liées entre elles pour ainsi dire par une solidarité circulaire, close » (Péguy, 1897a, p. 15, italique ajouté).

Si Walras met tous les échangeurs sur une ligne, Péguy affirme que la ligne et le nombre mathématique sont indéfinis alors que «le nombre social, économique, des hommes, des moyens, des produits, des travaux, est un nombre à chaque instant défini » (ibid., p. 15). Dans la réalité sociale, il existe des interrelations inévitables entre les individus - ainsi qu'entre leurs œuvres, leurs moyens et leurs produits de sorte qu'ils ne peuvent jamais être considérés comme des variables

23. En notant que dans le compte rendu sur Walras « on retrouve La Note conjointe et le temps abstrait », Thibaud considère que cela est « assez curieux » $(1975$, p. 140). 
totalement indépendantes les unes des autres, même si on les prend en compte tous et sans exception. Si Péguy semble vouloir souligner la difficulté de Walras à considérer mathématiquement les interdépendances au sein de son système, il propose de les prendre en compte à travers la fraction, laquelle représente à chaque fois I'individu comme faisant partie d'un tout. Walras sépare les individus au début de sa science, pour les réunifier plus tard dans l'État collectiviste (propriétaire des terres). Pour Péguy, cette réunification arrive trop tard et il est nécessaire de considérer les individus dans leurs interactions dès le début.

Walras cherche une solution parfaite à la question sociale, en posant le problème de « qui boira ce Château-Lafite » dans la société idéale et future (Walras, 1896). Péguy, pour sa part, se dit peu préoccupé par ce problème : «il est un peu étrange, vraiment, de tant s'inquiéter pour le château-Lafitte et pour sa future distribution, quand $c^{\prime}$ est pour le pain et pour l'éducation que s'engage par le monde la bataille sociale » (Péguy, 1897a, p. 17). Plus tard, dans De Jean Coste (1902), un texte crucial pour sa réflexion économique, Péguy affirme que la pauvreté constitue une limite économique fatale en dessous de laquelle pas une seule personne ne devrait se trouver dans une communauté humaine vraiment constituée (voir Bee, 2009 ; 2011), et il revient explicitement sur ce qu'il a dit à la fin du compte rendu sur Walras: "Je ne puis parvenir à me passionner pour la question célèbre de savoir à qui reviendront, dans la cité future, les bouteilles de champagne, les chevaux rares, les châteaux de la vallée de la Loire; j'espère qu'on s'arrangera toujours; pourvu qu'il y ait vraiment une cité » (Péguy, 1902, pp. 1033-1034, italiques ajoutés). Cinq ans après son compte rendu sur Walras, Péguy a toujours en tête de lui répondre lorsqu'il aborde directement les questions économiques. Ce qui confirme qu'entre-temps il ne s'est pas engagé sur une route qui n'a aucun rapport avec son compte rendu sur l'économie mathématique ; plutôt, il a continué à répondre à Walras. Si pour Péguy il ne s'agit pas d'imaginer une société idéale dans un temps indéfini, mais de s'immerger dans le temps économique pour le comprendre, c'est premièrement avec l'action qu'il entend lui répondre.

De ce point de vue, on comprend encore mieux ce qu'il veut dire lorsqu'il écrit à Walras que « les nécessités de l'action » risquent de ne pas lui laisser le temps de se consacrer davantage à l'économie mathématique. Le choix de l'action, aussi imparfaite soit-elle, signifie pour Péguy se plonger dans cette réalité hétérogène que Walras ne 
pouvait pas envisager pour des questions de méthode. L'action consistera dans un engagement pour l'éducation: ouvrir une librairie socialiste. Ce sera ensuite cette action qui lui suggérera comment penser une économie qui part de l'hétérogénéité des phénomènes économiques et ainsi comment trouver une réponse théorique au défi intellectuel posé par les travaux de Walras.

\section{Dans la communication économique universelle: la réponse de Péguy}

\subsection{Un retournement de méthode}

Comme il l'annonce dans sa lettre à Walras, Péguy abandonne la même année ses études à l'École normale supérieure, se marie et fonde, avec la dot de sa femme, la Librairie socialiste George-Bellais quelques mois plus tard, prélude aux Cahiers de la Quinzaine. De cette expérience, Péguy tire les premiers éléments pour mettre en place une éventuelle théorie économique. II présente ces éléments dans trois conférences tenues en 1904 à l'École des hautes études sociales, dans lesquelles on trouve une réponse théorique à la démarche initiée par Walras.

Cette fois-ci, la référence à Bergson est explicite et revendiquée. Péguy commence ses conférences en précisant que sa réflexion part d'une interprétation personnelle de la pensée du philosophe (voir aussi Devaux, 2000 ; Worms, 2000). Les conférences ont pour thème l'anarchisme politique et Péguy entend aborder la question du point de vue des études sociales.

Pour pouvoir débuter des études sociales, selon Péguy, il faut commencer par une étude de ce qu'il appelle la communication sociale. Par cette expression générale il désigne toutes les relations sociales allant de la guerre à l'« échange commercial de petit boutiquier à petit boutiquier » (Péguy, 1904b, p. 1806). La communication sociale a lieu entre ce qu'il appelle les personnes sociales. II peut s'agir aussi bien de personnes individuelles que de personnes collectives, d'administrations publiques ou privées, de coopératives, de sociétés de toutes sortes, jusqu'à l'alliance des peuples et de I'humanité elle-même. Péguy énumère longuement ces différentes typologies, précisément pour souligner leur hétérogénéité qui est donnée par les relations mutuelles (ibid., p. 1811-2). Ainsi, et en répondant également d'une certaine façon à Walras, il affirme : « II y aurait un très grand danger à définir les 
personnes sociales, avant d'avoir défini les communications sociales qu'il y a entre ces personnes. La tentation est en effet de prendre les personnes sociales [...] et d'étudier ensuite les communications qu'il y a ou qu'il peut y avoir entre ces personnes » (ibid., p. 1811). II est cependant plus approprié de se placer « au cours » de la communication entre ces personnes et de ne les définir qu'ensuite comme points de départ, d'arrivée ou de passage de cette communication (ibid.).

Plutôt que de partir d'individus homogènes et parfaitement libres de contraintes réciproques, comme le fait Walras, Péguy suggère de faire le contraire : partir précisément de ces contraintes, qu'il appelle servitudes ou pressions sociales, et ainsi d'étudier comment les personnes sociales s'interpénètrent les unes des autres. II s'agit pour Péguy d'un véritable « retournement de méthode » (ibid., p. 1820).

En ce sens, il suppose que lorsqu'il y a exercice d'autorité par la personne $A$ sur la personne $B$, on peut dire que le sens de la communication dans ce cas va de la personne $A$ à la personne B. Par conséquent, nous devons d'abord considérer la communication sociale, puis son sens et ensuite seulement les personnes sociales. Sinon, on risque seulement d'opérer « des sortes de coupes horizontales dans ce mouvement » et on s'en tient alors «à un examen de ces coupes » (ibid., p. 1809). On risque de perdre le mouvement de la communication et donc de ne traiter que des moments statiques, avec ces points qu'avec Bergson il appelle «points de repos» (ibid.). Chacun de ces points est en fait chargé de mouvement, de temps historique qui est perdu si on les considère comme indépendants les uns des autres. $\mathrm{Si}$ Walras part d'une concurrence homogène entre des individus égaux, Péguy part d'une concurrence telle qu'on la connaît en réalité, hétérogène et entre des individus inégaux qui exercent des pressions économiques les uns sur les autres.

Walras estime qu'il faut construire un idéal dans lequel les contraintes et l'hétérogénéité n'existent pas et ensuite - comme il le soutient dans un passage cité par Péguy dans son compte rendu - agir « comme si cela pouvait et devait avoir lieu » (Walras, 1896, cité par Péguy 1897 a, p. 13). Mais cela signifie théoriser une «liberté imaginaire ", comme celle de la Déclaration des droits de l'homme et du citoyen (Péguy 1904b, p. 1813) ${ }^{24}$. Pour Péguy, en revanche, les problèmes de liberté sont essentiellement des problèmes de libération

24. Dans la Déclaration des droits de l'homme et du citoyen, Péguy entrevoit « un effort constant pour fabriquer des hommes séparés, des individus sporadiques sans communications les uns avec les autres » (1904b, p. 1814). 
et c'est «la force de libération d'un organisme quelconque » qui «mesure sa valeur sociale» (ibid., p. 1823). Cette force de libération est liée à la capacité d'une institution à absorber les pressions sociales. Elle peut être évaluée seulement quand l'absence de pressions n'est pas supposée par hypothèse et sa transmission est donc précisément l'objet d'étude.

\subsection{La transmission de la pression}

Pendant qu'il prépare ses trois conférences, données en janvier 1904, Péguy rédige aussi un texte - écrit en décembre 1903 et publié en janvier suivant - dans lequel il explique la situation économique des Cahiers. II a retiré une partie de ce texte avec l'intention évidente de le publier plus tard ; mais comme c'est souvent le cas, ce texte n'a jamais été parachevé, ni publié ${ }^{25}$. Néanmoins, on y trouve un approfondissement exceptionnel de la pensée économique de Péguy que I'on ne perçoit alors qu'en filigrane dans plusieurs autres textes, ainsi que plus explicitement dans les conférences de 1904.

Dans ce texte, Péguy affirme qu'« une simple inspection de toute la réalité » nous dit que dans le monde économique il existe une «communication économique universelle» (1903, p. 1280, italique ajouté). Suivant encore une fois l'approche bergsonienne, Péguy nous invite à prendre en compte dès le départ qu'aucune entreprise n'est en « repos », mais qu'elle est toujours en mouvement et que son mouvement communique avec «l'immense mouvement économique universel » (ibid., p. 1279):

Telles seraient les données de ce problème économique extrêmement important, le problème de la pression, de la servitude économique : baignant de toute parts, ou d'un grand nombre de parts, qui seraient à déterminer, dans le mouvement, dans la vie économique universelle, toute entreprise économique reçoit, transmet, exerce des pressions, des servitudes économiques; la discussion, au moins sommaire, de ce problème, serait aisée, comme il advient, au moins en commençant, pour les problèmes économiques. (Péguy, 1903, p. 1279-80)

En étudiant comment ces pressions sont transmises de toutes parts, on peut éviter que les responsabilités sociales ne soient mal réparties. Par exemple, une entreprise capitaliste ne peut être considérée comme créant de toutes pièces la pression économique qu'elle transmet sur ses 
travailleurs que si elle $n^{\prime}$ est pas considérée comme subissant constamment une forte pression de la part des consommateurs. En réalité, toute entreprise «reçoit par en haut, des consommateurs, de ses clients, de ses acheteurs une pression économique, une servitude qu'elle transmet à ses ouvriers, aux producteurs, à ses vendeurs, et qu'en dessous elle exerce sur eux » (ibid., p. 1276). Il n'est pas correct d'évaluer une entreprise - qui baigne inévitablement dans le «temps économique » (Péguy, 1905, p. 262) - comme s'il s'agissait d'une entreprise fictive, opérant dans un temps imaginaire, purement abstrait. Chaque entrepreneur, en réalité, n'est rien d'autre que cela :

un homme qui porte d'une valeur déterminée à une valeur déterminée cette pression économique, cette servitude ; c'est un homme qui porte de $V$ à $V^{\prime}$, de $P$ à $P^{\prime}$ une pression économique; il reçoit une pression égale à $P$; il transmet, il exerce une pression égale à $P^{\prime}$. (Péguy, 1903, p. 1278)

Afin d'évaluer une entreprise sur le plan économique et social, il faut mesurer la différence entre $\mathrm{P}$ et $\mathrm{P}^{\prime}$. Si $P^{\prime}>P, c^{\prime}$ est-à-dire si la pression transmise est supérieure à celle reçue, alors c'est une entreprise qui mène ses ouvriers dans la misère " par une mauvaise administration industrielle et commerciale, par l'autorité de commandement patronal » (1903, p. 1787). Si $P^{\prime}=P$, alors il s'agit d'une entreprise économiquement et socialement neutre. Si, en revanche, $P^{\prime}<P$, cela signifie qu'il s'agit d'une entreprise économique et sociale, socialement bonne. Plus elle absorbe la pression, plus elle marque un progrès. C'est ce que Péguy considère comme étant à proprement parler une entreprise socialiste. Ce qui mesure la valeur d'une entreprise n'est pas la totalité de la servitude économique qu'elle transmet, mais la part de cette servitude qui lui « revient en propre » $(1903$, p. 1277 ; voir aussi 1904b, p. 1822) :

la valeur économique, sociale de l'entreprise, de l'œuvre, de l'usine, de l'atelier, se mesure à la valeur de cette transmission même, à la qualité, à la souplesse, à la fidélité, à l'économie de la transmission. (Péguy, 1903, p. 1276)

La prise en compte du mouvement nécessite une évaluation de la qualité de la transmission, c'est-à-dire des différents modes d'organisation, de la souplesse dans la gestion, de l'attention constante à cette transmission, qui conduisent une entreprise à absorber la pression dans la mesure du possible. Considérer l'entreprise comme "isolée ", seule, sans communication, c'est en avoir une image «parfaitement statique », de «manuel » (1903, p. 1279), et dans ce cas on ne peut 
que considérer la pression totale qu'elle génère à partir de ce « point de contact », de cette coupure du mouvement (1904b, p. 1821).

Le manque d'attention pour la transmission de la pression économique peut conduire à considérer les entreprises capitalistes comme les seuls producteurs de pression et donc à imaginer qu'une organisation socialiste puisse miraculeusement la dissoudre complètement sans en produire de nouvelles. Mais il est commode et aussi totalement illusoire de croire qu'une organisation socialiste puisse absorber toute la pression sociale : "cette singulière opération économique ne ferait pas un miracle, elle ne serait pas merveilleuse, elle ne serait pas mystérieuse, elle ne serait pas miraculeuse; elle ferait un surmiracle, elle serait surmerveilleuse, sous-mystérieuse, surmiraculeuse; et quand on a éloigné cette hypothèse des derniers confins de l'imagination même, on s'aperçoit avec stupeur qu'elle règne généralement dans les esprits, et qu'elle est tout l'étatisme » (1903, p. 1282, italique ajouté).

De même, il serait irréaliste d'imaginer le cas « d'une entreprise économique ne recevant aucune pression, aucune servitude économique et créant toute la pression, toute la servitude économique d'un monde; c'est à peine si les pires tyrannies de l'Orient, de l'ancien Orient peuvent donner quelque image extrêmement éloignée d'une telle situation économique » (ibid., pp. 1281-2). Tout aussi irréelle serait la situation dans laquelle une organisation socialiste serait capable d'absorber complètement ne serait-ce qu'une seule des pressions qu'elle reçoit; ainsi que la situation dans laquelle une entreprise capitaliste serait capable de produire ne serait-ce qu'une seule pression économique à partir de rien. C'est plutôt entre ces deux limites que se produit la transmission de toutes les servitudes économiques et sociales et que les pressions peuvent être partiellement absorbées. II s'agit alors de savoir comment une organisation peut absorber la pression.

\subsection{Autorité de commandement et autorité de compétence}

Péguy est conscient de la difficulté de présenter une théorie qui veut se « modeler » sur la réalité économique dont il parle; c'est pourquoi se différenciant une fois de plus de Walras qui propose une solution mathématique à la question sociale - il déclare dans ses conférences de 1904 : « C'est plutôt un problème qu'il faut présenter qu'une solution que l'on apporte » (1904b, p. 1815).

Le problème est que lorsqu'il y a communication, il y a exercice d'autorité. On serait donc amené à penser que pour résoudre le 
problème, il suffirait d'éliminer la communication sociale. Cependant, l'absence de communication signifie la mort sociale : « si on supprimait le négoce, le travail, les relations d'atelier et de marchandises, I'humanité n'existerait plus demain matin » (ibid., p. 1815). Le problème peut donc être formulé en ces termes : étant donné que la communication sociale n'est rien d'autre que la transmission de servitudes, comment réduire le plus possible cette transmission en réduisant le moins possible la communication?

Le problème paraît insoluble (voir Thibaud, 1978), si l'on ne tient pas entièrement compte du fait qu'il existe en réalité au moins deux formes «hétérogènes » d'autorité, à la définition desquelles Péguy consacre une grande partie de la première conférence de 1904. II appelle la première forme d'autorité " arché », pour indiquer ce qui est au début, ce qui vient en premier, ce qui initie, l'origine. Et il appelle la deuxième forme d'autorité "cratos", pour indiquer l'exercice d'un pouvoir pur et simple obtenu par la force. Cette distinction permet d'éviter cette confusion «militaire » qui conduit le guide à prendre le commandement de l'équipe, pour le simple fait de s'être trouvé comme premier à marcher et qu'on se mit à le suivre (Péguy, 1904b, p. 1803). Ces deux formes d'autorité se distinguent avant tout par un rapport différent qu'elles entretiennent avec la liberté. Le cratos, que Péguy appelle autorité de commandement, est fondé sur la négation violente de la liberté d'autrui ; tandis que l'arché, que Péguy appelle autorité de compétence, est fondé sur la liberté d'autrui. Par exemple, lorsque les parents ne peuvent pas s'occuper de l'éducation de leurs enfants, ils s'en remettent à la compétence d'un enseignant. II «fait autorité » à leurs yeux et à ceux de leurs enfants. Mais si l'enseignant profite de son rôle pour imposer certaines idées ou lectures aux élèves, il agit alors comme un « autoritaire » (ibid., 1818-9).

Il existe donc deux formes de liberté liées à ces deux formes d'autorité. Une liberté de désobéissance est exercée à l'égard de l'autorité de commandement. C'est ce à quoi Péguy fait référence avec le mot «acratie », c'est-à-dire une liberté qui s'oppose directement à une autorité de gouvernement, donc une liberté politique. Par contre, une liberté de conscience ou liberté d'examen s'exerce à l'égard de l'autorité de compétente, c'est-à-dire « des libertés de récusation, ou de dégagement » (ibid., p. 1805). C'est ce que Péguy appelle " anarchie », qui est une "liberté de retraite » une "liberté de fuite plutôt qu'une liberté d'opposition »; c'est la liberté que l'on peut avoir envers une administration, donc il s'agit proprement d'une liberté 
économique. Adoptant toujours l'approche bergsonienne, revendiquée au début de la conférence, Péguy explique que la différence entre les deux autorités est donc celle-ci :

l'autorité de compétence a ce caractère propre que, tandis qu'on se révolte contre l'autorité de gouvernement par un mouvement direct, au contraire on ne peut convenablement se révolter contre l'autorité de compétence qu'en déclinant la compétence, par un déclinatoire de compétence; par ces seules différences nous voyons combien est hétérogène le classement usuel établi entre les autorités. (Péguy, 1904b, p. 1805)

Pour pouvoir exercer la liberté de dégagement, il est nécessaire de ne pas être soumis à une autorité de commandement qui l'empêche. La liberté qui sous-tend l'autorité de compétence exige que l'autorité de commandement soit réduite le plus possible. Si une administration est imposée de manière autoritaire, elle ne peut pas faire autorité.

En posant le problème des études sociales en ces termes nouveaux, Péguy indique une solution. C'est dans ce sens, en effet, qu'il est possible de penser à une réduction de la transmission de la servitude qui ne se traduit pas forcément par une réduction de la communication, mais plutôt par une communication plus libre. II s'agit de réduire autant que possible l'autorité de commandement, sans pour autant réduire l'autorité de compétence :

une communication sociale est une communication sociale libre quand : $1^{\circ}$ il ne $s^{\prime} y$ exerce aucune autorité de commandement ; $2^{\circ}$ quand les autorités de compétence qui s'y ajoutent se font équilibre. (Péguy, 1904b, p. 1820)

Réduire l'autorité de commandement à zéro ne signifie pas réduire la communication sociale à zéro, car elle peut reposer sur des autorités de compétence qui s'équilibrent mutuellement. C'est à ce point des conférences, et seulement ici, que Péguy utilise l'expression « échange social » comme synonyme de « communication sociale ». Reprenant ce qui a été dit précédemment, il affirme que pour qu'il y ait vie sociale il faut qu'il y ait « sans cesse communication sociale, échange social » et qu'à chaque fois qu'il y a communication sociale il y ait «échange d'autorité » (ibid., p. 1820). Si l'idée de l'échange d'autorité apparaît ici, c'est parce que Péguy pense à l'échange d'autorité de compétence ; un échange qui peut consister en un équilibre précisément lorsque l'exercice de l'autorité de commandement a été quasiment supprimé. Une fois libéré, pour Péguy l'échange n'est donc pas un rapport de force, mais un équilibre d'arché. 
Walras part de l'hypothèse d'individus qui échangent dans la libre concurrence et pour y parvenir il identifie un État capable d'absorber toute la pression sociale. Péguy, en revanche, part d'une concurrence hétérogène donnée et, pour arriver à un échange libre, identifie un processus de libération des autorités de commandement au sein de l'entreprise.

Dans sa Cité socialiste (1897b), publiée quelques mois après le compte rendu sur Walras, Péguy parle d'une communauté dans laquelle le gouvernement des hommes est remplacé par l'administration des biens. Dans ses conférences de 1904, il explique en quoi consiste plus précisément ce mouvement de libération dans I'ordre politique et économique : il s'agit de créer des organisations économiques proprement socialistes, dans lesquelles il y a une liberté politique et économique, c'est-à-dire dans lesquelles il n'y a pas de commandement sur les hommes, ce qui laisse toujours la possibilité de récuser l'autorité de ceux qui administrent les biens. C'est ce type d'administration que Péguy a expérimentée avec ses Cahiers.

\section{Les Cahiers de la Quinzaine : une entreprise économique exemplaire}

\subsection{Au-delà de la forme juridique}

Pris par les débuts de l'affaire Dreyfus, Péguy ne prête pas une attention particulière aux finances de sa librairie socialiste, qui deviennent rapidement fragiles ${ }^{26}$. Des amis lui viennent en aide pour soutenir son initiative et créent avec lui, en 1899, la Société Nouvelle de Librairie et $d^{\prime}$ Édition, qui absorbe le capital de la Librairie George-Bellais et engage Péguy comme délégué à l'édition. Le conseil d'administration est composé de personnalités du mouvement socialiste, dont Lucien Herr, Léon Blum et François Simiand. Avec Jean Jaurès, ils font partie des partisans de I'unification de ce mouvement en France. Un signe de cette unification est le congrès socialiste de décembre 1899, au cours duquel il est établi que la presse socialiste ne doit pas publier de positions critiques à l'égard du mouvement ou de ses franges. Explicitement contre cette décision et pour un socialisme non autori-

26. La librairie, souvent attaquée par des centaines de manifestants antidreyfusards, servait de siège aux étudiants dreyfusards du Quartier Latin, d'où ils partaient pour défendre les professeurs de la Sorbonne attaqués à leur tour, voir Leroy (2014, p. 82-3), voir aussi Halévy (1918, p. 48). 
taire, Péguy décide de créer des cahiers bimensuels pour publier librement des positions critiques au sein du mouvement socialiste. Le conseil d'administration de la Société Nouvelle s'oppose fermement à ce projet et Péguy n'a d'autre choix que de démissionner pour créer ses Cahiers quelque jours plus tard, le 5 janvier 1900. Son capital initial reste bloqué dans la Société Nouvelle et il ne peut le récupérer que petit à petit. Cette naissance traumatique des Cahiers conduit Péguy à les considérer comme une entreprise socialiste très différente de la précédente. De cette nouvelle expérience, il va tirer les éléments pour esquisser la théorie économique qu'il approfondi dans le texte de 1903 et explique dans les conférences de 1904.

Pour ceux qui assistent à ces conférences, en fait, les références implicites à ces événements sont évidentes, d'autant plus évidentes que Péguy a beaucoup écrit à leur sujet. En janvier 1901, à peine un an après la fondation des Cahiers et la rupture avec le conseil d'administration de la Société Nouvelle, Péguy publie ses souvenirs de ces moments :

Je me rappellerai toujours, pour l'administration de ma vie, de quel ton Herr vous dit: Nous somme unanimes à penser que vous allez marcher contre tout ce que nous avons fait ensemble. Nous sommes unanimes à n'accepter pas cette publication. - Ils étaient unanimes! Et qu'est-ce que cela prouve ? Esprits à peu près identiques, ayant la même culture, les mêmes bonnes et les mêmes alourdissements, ces cinq administrateurs étaient plus facilement unanimes entre eux que je ne suis unanime avec moi. Quand Herr discute avec Simiand il y a moins de profonde variété, moins de pénible et douloureuse incompatibilité que quand je discute avec moi. C'est dire qu'il y a dans leurs assemblées moins de véritable discussion que quand je $\mathrm{m}^{\prime}$ assemble tout seul.

(Péguy, 1901a, p. 130)

Péguy conteste qu'il suffise de dire nous sommes unanimes pour considérer «que I'on a tout dit»; il en va de même pour le nous sommes en majorité (1901a, p. 130). En réalité, il s'agit de « constatations de quantités » qui risquent d'empêcher une écoute scrupuleuse de la voix de la raison. L'attitude consistant à vouloir «majoriser » le voisin n'est non seulement pas un esprit révolutionnaire, mais représente l'envahissement de la vie sociale par la vie politique (ibid.). Si Péguy n'a rien contre le fait que certains dimanches, le citoyen aille voter pour un autre citoyen dans un conseil municipal ou législatif, ce qui le dérange, c'est que ce mode, typique de la « votation parlementaire bourgeoise ", a également contaminé l'administration de la vie sociale : «le citoyen qui, son bulletin mis, rentrant à la maison, dirait à 
sa femme: à présent nous allons voter pour savoir si nous ferons ce soir un pot au feu me semblerait un dangereux maniaque. Pourtant c'est là que nous en sommes » (ibid., 131).

Péguy soutient que la question sociale ne peut pas être résolue « par quelque merveilleuse constitution, mais par une organisation patiente du travail, de l'économie, des ateliers, de la production, de la consommation » (1904, p. 1815). De son côté, cette organisation économique patiente ne peut se résoudre tout simplement dans un merveilleux règlement qui établit comment prendre les décisions, par exemple par un vote à la majorité. Les règlements et les statuts sont comme les constitutions et les lois : la plupart sont « statiques, faites pour le tracé, pour le repos et non pas pour le mouvement, la vie, le travail »; ils sont faits par des hommes, dit Péguy, qui sont trop juristes et pas assez économistes (ibid., p. 1809). L'expérience de la Société Nouvelle, $\mathrm{d}^{\prime}$ ailleurs, le lui a appris à ses dépens ${ }^{27}$. La société était conçue comme une «coopérative de producteurs et de consommateurs » (Péguy, 1901 a, p. 118), mais au moment venu une autorité de commandement a été exercée, bien que de manière unanime. Qu'une décision soit imposée par la force quantitative de l'unanimité ou de la majorité, cela n'enlève pas qu'elle soit fondée sur le cratos et non sur l'arché, c'est-à-dire sur la subordination au commandement plutôt que sur la libre reconnaissance des compétences. L'organisation patiente du travail vise à réduire la pression, alors que dans ce cas-ci, la coopérative de producteurs et de consommateurs l'a augmentée.

Péguy conclut ses conférences de 1904 en disant que la révolution sociale ne peut pas consister simplement à remplacer les entreprises capitalistes par des entreprises socialistes, car cela pourrait se faire en remplaçant les entreprises capitalistes qui fonctionnent bien par des entreprises socialistes qui fonctionnent mal. Le public de ces leçons sait bien que lorsqu'il parle d'entreprises socialistes mal gérées, il pense à la Société Nouvelle ${ }^{28}$.

27. Dans le texte de décembre 1903, Péguy précise qu'il avait connu «malheureusement » des entreprises socialistes mal administrées pendant les « dernières années » (1903, p. 1278).

28. D'autre part, les auditeurs s'attendaient probablement à entendre Péguy parler de l'expérience des Cahiers, sachant qu'il était évidemment entendu en tant que leur gérant. Le lendemain de la première conférence, Georges Sorel écrivit à Péguy en lui disant que les auditeurs avaient trouvé qu'il avait raconté « des choses inintelligibles, subtiles et sans rapport avec le sujet » et que cela pourrait avoir des conséquences « grave pour l'avenir des Cahiers» (Sorel, 1904). Sorel, donc, suggère que Péguy soit à l'avenir plus clair, ce qu'il tâcha de faire : dans les deuxième et troisième conférences, Péguy s'est en effet appuyé sur plus d'exemples concrets. 
Grâce à cette expérience néanmoins, Péguy fait un pas important dans sa réflexion : ce qui compte dans l'évaluation de la valeur sociale d'une entreprise n'est pas sa forme juridique mais sa gestion, c'est-àdire la manière dont elle répartit les pressions au sein de l'économie de transmission. Toute entreprise, qu'elle soit capitaliste ou socialiste, opère dans la communication économique universelle et doit être évaluée dans cette perspective : "peu importe à cet égard que les entreprises termes de comparaison soient ou ne soient pas intérieurement capitalistes, socialistes, communistes » (Péguy, 1903, p. 1277, italique ajouté). Les entreprises économiques «collectivistes, socialistes, communistes » qui augmentent, transmettent ou de toute façon absorbent moins la pression économique «par une administration économique mauvaise, par l'abus du gouvernement » sont en réalité « des instruments de régression économique » (ibid., p. 1278).

Au contraire, bien qu'il puisse y avoir « des patrons qui accroissent odieusement, monstrueusement la pression économique ", il y en a aussi d'autres qui non seulement ne l'augmentent pas, mais l'absorbent «quelquefois beaucoup » (ibid.). Une institution communiste qui accroît la pression, fait « littéralement, exactement du travail bourgeois, de l'action antisocialiste", alors qu'une institution non communiste qui absorbe de la pression fait «littéralement, exactement, du travail, de l'action socialiste » (ibid.). Lorsque Péguy parle d'une entreprise qui, sans être communiste, est néanmoins attentive, fidèle à l'économie de la transmission de la pression et l'absorbe «par de la bonne administration, par de la bonne gérance» (1904b, p. 1823, italique ajouté), il pense certainement aussi et surtout aux Cahiers. D'ailleurs, déjà en 1901 il écrit qu'avec Pages libres, les Cahiers " sont de toutes les institutions socialistes, révolutionnaires, libérales, républicaines, la plus viable » (1901b, p. 1654, italique ajouté).

\subsection{Retirer le pouvoir au lieu de le partager}

Avec les Cahiers, Péguy entend trouver un moyen d'éviter de se retrouver à l'avenir dans une situation similaire à celle de la Société Nouvelle. En d'autres termes, il veut prouver la viabilité d'une entreprise qui absorbe la pression sociale en réduisant autant que possible l'autorité de commandement exercée par quiconque est en communication avec elle, aussi bien ceux qui l'ont créée que ceux qui fournissent le capital, y travaillent ou consomment ses produits. Cela l'amène à vouloir renoncer à l'autorité de commandement sur ses collaborateurs, ce qu'on lui reconnaît à juste titre (Riquier, 2017 ; Vitry, 2015 ; Leroy, 
1975). Mais c'est surtout le fait de ne pas avoir à la subir ce qui peut précisément lui permettre de ne pas l'exercer. Par conséquent, si l'on sait que Péguy a évité d'exercer une autorité de commandement dans les Cahiers, un examen plus approfondi est nécessaire pour voir quelles précautions il a pris pour ne pas la subir. Celles-ci font toute l'originalité de son entreprise, ce qui n'a finalement rien à voir avec le fait qu'il puisse être considéré comme un «marginal » qui ne veut pas jouer selon les règles démocratiques (Leroy, 1975, p. 29).

Pour Péguy, il s'agit de briser la chaîne pour laquelle « chacun rend à celui qui vient l'abus d'autorité que, venant lui-même, il a subi de celui qui s'en va» $(1903$, p. 1788). C'est pour cela que : " premièrement nous devons rigoureusement nous interdire de subir aucun entraînement ; nous devons nous libérer des entraînements comme de toutes les autres servitudes; deuxièmement nous devons rigoureusement nous interdire d'exercer aucun entraînement » (ibid.).

Dans le cas des Cahiers, Péguy veut s'empêcher, en tant qu'initiateur et administrateur, d'exercer une autorité de commandement sur les collaborateurs, les abonnés et les lecteurs et empêcher que tous, à leur tour, exercent une autorité de commandement les uns sur les autres ou sur lui en tant qu'administrateur. En ce sens, Péguy ne se considère jamais comme le «directeur » d'une revue, mais il y tient toujours à dire qu'il est le « gérant » des Cahiers. Ce choix n'est pas une coquetterie mais a des fondements politique et économique majeurs. II est gérant au sens où il est le garant de l'économie de la transmission : c'est-à-dire, celui qui est « au centre des communications » et qui administre les biens afin d'équilibrer au mieux les autorités de compétence, en absorbant le plus possible l'autorité de commandement dans et sur les Cahiers, « une autorité de commandement que personne jamais, ni l'unanimité des abonnés, ni les auteurs, ni le gérant n'y exercent » (Péguy, 1902a, pp. 163-169). ${ }^{29}$ De son point de vue, il ne s'agit donc pas de partager l'autorité de commandement avec le plus grand nombre de personnes possible pour décider à la majorité, voire à l'unanimité. II s'agit, plutôt, dans la mesure du possible, de le refuser à tous y compris à lui-même. II ne s'agit pas de donner plus de pouvoir possible à tout le monde, mais de le retirer le plus possible à tout le monde.

29. L'absence de publicité dans les Cahiers doit également être lue sous cet angle, la publicité étant une forme d'entraînement tant pour les consommateurs que pour les producteurs (voir Péguy, 1897b). 
Dès le départ, l'administration expérimentée par Péguy a pris cette orientation. Bien qu'il ne dispose pas d'un capital initial, il ne cherche pas de partenaires pour cette nouvelle initiative. Ceux qui remplacent la figure des actionnaires dans sa société sont les « souscripteurs réguliers et extraordinaires", ce que Péguy appelle les «auteurs économiques» (1904a, p. 208). Ce sont ceux qui décident de ne pas décider, c'est-à-dire ceux qui décident d'apporter une contribution afin que personne, y compris eux-mêmes, ne puisse exercer un quelconque contrôle sur l'organisation. C'est en ce sens que sur la deuxième de couverture des Cahiers est écrit dès les premiers numéros : «la souscription ne confère aucune autorité sur la rédaction ni sur I'administration : ces fonctions demeurent libres ».

Malgré tout, Péguy est souvent confronté à des abonnés qui, tout en appréciant le travail des Cahiers, voudraient que certains de leurs collaborateurs cessent de publier parce que cela ne correspond pas à leurs vues. À un moment donné, Péguy aborde le problème de cette manière : "Je révèle ici un secret de ma gérance : Tous les cahiers, sans aucune exception, les jaunes et les blancs, sont faits pour mécontenter un tiers au moins de la clientèle » (1902a, p. 169).

On pourrait, donc, penser que Péguy traitait les Cahiers comme une institution qui devait profiter aux producteurs plutôt qu'aux consommateurs (Thibaud, 1975, p. 143-4). En réalité, la modalité du tiers mécontent - si l'on peut l'appeler ainsi - n'est pas faite uniquement pour préserver la liberté des collaborateurs, en ne cédant pas à la censure des lecteurs et donc à leur autorité de commandement sur la rédaction. II est également fait pour préserver les lecteurs eux-mêmes. En fait, Péguy explique que l'important dans ce mode d'administration est que le tiers mécontent ne soit pas toujours le même. Comme tout le monde n'apprécie pas les mêmes cahiers, satisfaire certains en ne publiant pas ce qui les dérange reviendrait nécessairement à s'empêcher de satisfaire les autres. Permettre aux deux tiers d'être satisfaits à chaque fois n'est possible que parce que la fois suivante, le tiers insatisfait n'est pas le même que la fois précédente et fait donc partie des deux tiers satisfaits. C'est ainsi que Péguy tente de repartir et donc de réduire la pression que les lecteurs exercent les uns sur les autres.

Au contraire, pour Péguy, céder à une telle pression reviendrait à se comporter comme une revue qui suit la mode ou une ligne de parti, c'est-à-dire une revue qui satisfait tout le monde ou où tout le monde est toujours d'accord. Alors que mécontenter c'est « heurter, remuer, 
faire travailler » (Péguy, 1902a, p. 169), c'est encourager la formation $d^{\prime}$ 'un esprit de curiosité, d'étonnement, et non pas s'asseoir sur un « esprit mort de statique » sur une sorte $d^{\prime}$ '«autointoxication par complaisance mutuelle, un empoisonnement par la respiration d'un air confiné en chambre close d'admiration perpétuelle » (ibid., p. 169). Les Cahiers sont un espace éminemment libre de rencontre et de débat, ils ne sont pas un instrument de propagande. S'ils devenaient une revue qui fait de l'entraînement (pour lui, la pire forme d'autorité de commandement), Péguy affirme qu'il serait le premier à s'y opposer et que ce serait la meilleure bataille qu'il pourrait mener pour la liberté d'esprit.

Péguy publie des textes qu'il ne partage pas, ce qui «prouve que, intellectuellement, nos différents cahiers sont, dans les séries, parfaitement libres » (1904c, p. 1283). Ainsi, le gérant se traite, en tant que lecteur lui aussi des Cahiers, comme il traite tous les autres lecteurs. II en va de même pour lui-même en tant qu'auteur et il "se traite, comme auteur, comme il traite les autres auteurs»: «À peine ai-je besoin de dire que je ne m'accorde, comme auteur, aucun avantage. Ma copie attend comme les autres copies » (Péguy, 1902a, p. 170). Le concept est clair : celui qui gère, gère au nom de tous, $y$ compris de luimême (ibid., 170) ${ }^{30}$.

\subsection{Les inventions administratives pour une gestion ouverte}

Déjà après quelques années, les Cahiers souffrent de leur succès. Ils sont distribués et lus dans le reste de l'Europe et au-delà. Cet espace intellectuellement libre accueille de plus en plus de textes écrits par un seul auteur, avec un nombre de pages variable et souvent supérieur à celui initialement prévu, devenant ainsi une sorte de maison d'édition qui imprime et diffuse un livre tous les quinze jours. Le travail éditorial, typographique et administratif est important. Dans l'ensemble, le nombre de pages imprimées augmente, donc les coûts, tandis que le

30. Péguy écrit à ce propos : «Un bon tiers de nos abonnés insiste pour que j'écrive plus souvent dans les cahiers. Je résiste à ce tiers comme aux autres. Je ne m'accorde d'écrire que quand j'ai assuré le travail de gérance et le travail de fabrication » (1902a, p. 170-1). Et il ajoute : « Je passe un grand tiers de mon temps à l'établissement industriel des cahiers, à la correction des épreuves. [...] Je passe un petit tiers de mon temps à l'établissement commercial des cahiers, à la gérance. [...] Le tiers qui me reste, j'écris. [...] Je suis un pauvre industriel et, comme on dit, un petit boutiquier. Je le suis parce que je dois l'être. Je suis heureux de l'être. J'exerce avec passion deux métiers réels. Ces deux métiers m’ont appris sur les réalités économiques, politiques, morales, et sociales, plus que ne m'avaient enseigné en cinq ans mes maîtres et mes sous-maîtres. [...] Je suis un économe, un gérant, un intendant, un cuisinier, un employé, un commis » (ibid., pp. 171-3). 
prix de l'abonnement annuel ne varie jamais, afin de le rendre accessible même aux plus démunis. L'un des collaborateurs les plus importants, Romain Rolland, dira des Cahiers : «Qu'ils aient pu, en cinq ans, conquérir ce public de choix, en dépit des efforts faits pour les étouffer par le parti socialiste et une part de l'Université, témoignait, chez le 'gérant', $d$ 'une rare astuce unie à la ténacité, et du dévouement de ses fidèles » (1946, pp. 330-331, italiques ajoutés). Lorsqu'en raison de son succès, l'entreprise a besoin d'un soutien financier supplémentaire, Péguy conçoit quelque chose qui doit être à nouveau compris comme tentative de réduire l'autorité de commandement que les parts de capital nécessaires auraient pu avoir sur les Cahiers et donc sur ses collaborateurs, ses lecteurs et son administration : un prêt. Dans ce cas, l'astuce - ou "l'invention administrative », comme Péguy appelle ses expérimentations (1905, p. 263) - réside dans la manière dont les conditions du prêt ont été établies. Cette expérimentation - tout comme celles du tiers mécontent ou des souscripteurs à la place des actionnaires - peut sembler sans importance, mais elle ne l'est certainement pas pour Péguy, qui a le «don génial [...] de faire surgir du moindre incident de boutique les considérations morales les plus hautes et les plus inattendues, avec d'amples développements d'une valeur d'art et de pensée exceptionnelle » (Rolland, 1946, p. 85). ${ }^{31}$ Péguy explique et revendique précisément les termes de ce prêt dans son article de décembre 1903, publié en janvier 1904, dont il extrait le texte sur la transmission des pressions économiques analysées jusqu'ici, qui constitue donc le développement théorique de cette explication économique.

Péguy prétend avoir poussé à la limite certaines conditions du prêt habituel afin d'en faire un cas particulier : le prêt des Cahiers. Il dit, en effet, avoir « reporté à l'infini l'échéance du remboursement, qui tend à s'éloigner ", et avoir « réduit à zéro le taux d'intérêt, qui tend à diminuer » (1904a, p. 209). Ainsi, en réalité, les créanciers deviennent de fait des actionnaires qui détiennent des parts du capital. Pour garantir ce capital, Péguy affirme avoir pris soin de maintenir l'avoir des Cahiers à un montant au moins égal à la valeur totale du prêt. Cependant, contrairement aux actionnaires classiques, non seulement les créanciers du prêt des Cahiers ne réalisent aucun bénéfice, même s'ils supportent le risque de perte, mais surtout ils n'ont aucun pouvoir de

31. Comme le dit Latour (2014, p. 23), Péguy «n'a fait que penser, comme Bergson, le même problème partout ». 
décision. Les trois cinquièmes du prêt demandé sont trouvés, de sorte que l'opération est en grande partie réussie. Connaissant tous les souscripteurs du prêt, Péguy déclare que les seuls qui n'ont pas souscrit sont ceux qu'il appelle les demi-bourgeois. Les plus pauvres et les grands capitalistes ont donné leur contribution, tandis que ceux qui se sont abstenus sont ceux qui ne peuvent pas accepter de donner de l'argent en perdant du pouvoir au lieu d'en obtenir. En fait, même dans ce cas, l'objectif de Péguy est précisément de mettre en communication les abonnés, les collaborateurs et les lecteurs en général, dans un équilibre qui minimise la maîtrise que le capital pourrait exercer sur eux tous, en lui retirant du pouvoir.

C'est ainsi que Péguy, en décembre 1903, décrit, dans son style coutumier, l'administration des Cahiers comme une entreprise économique qui communique de tous côtés :

étant données les disponibilités, les propositions du marché du travail, étant donné ce qui existe, étant donné ce qui se propose, étant donnés les dévouements, de travail, qui se présentent, libres, librement, étant donnés les travaux faits ou à faire, libres, librement, étant donnés les auteurs, étant donnés les ouvriers, étant données les œuvres, étant données les collaborations, étant données toutes les propositions et toutes les réalisations de travail, et d'autre part, en face, étant donnés les abonnés, qui ne sont pas moins libres, étant donné le public, au moins éventuel, étant donné le peuple, au moins en espérance, notre office est d'administrer du mieux que nous pouvons la communication du travail entre les premiers et les deuxièmes éléments. (Péguy, 1903, p. 1792)

Cette « méthode » qui est « d'administration et de travail » est différente de la méthode démagogique non seulement pour une question de degré, mais aussi et surtout pour une question de nature :

L'entraîneur sollicite ; il arrache ; il extorque ; il va chercher ; l'administrateur, au contraire, attend ; il table sur la situation économique donnée ; il part uniquement des propositions de travail spontanés, libres; et tout son effort tend à ce que par une administration entendue ces propositions aboutissent à donner le plus du rendement le meilleur. (Péguy, 1903, p. 1792)

Pour Péguy, l'entreprise qui fait de l'action socialiste n'est pas celle qui a la gestion la plus partagée possible - dans laquelle il y a le plus $d^{\prime}$ individus possible pour décider - mais celle qui pratique une gestion aussi ouverte que possible à toutes les propositions qui se présentent spontanément, afin que chacune d'entre elles puisse s'épanouir au mieux. Gérer autrement implique la possibilité de faire d'autres choses et pas simplement les mêmes choses d'une manière qui n'est que quan- 
titativement différente de la précédente. L'objectif est de surmonter la subordination typique de la direction capitaliste sans céder à la tentation facile de la direction collectiviste. Il s'agit, en réalité, d'une administration qui garantit qu'aucune direction ne se mette en place :

II n'y aura jamais pour nous aucune relation d'auteur à directeur, d'employé à employeur, aucune subordination, mais corrélation d'homme libre à homme libre, d'auteur à gérant sans intermission commerciale d'autorité bourgeoise. L'auteur écrira sous sa responsabilité personnelle sincèrement et librement, vraiment. II $\mathrm{n}^{\prime}$ engagera pas le prochain. Le prochain ne l'engagera pas. II n'engagera pas l'administration des Cahiers. L'administration des Cahiers ne l'engagera pas. (Péguy, 1900, pp. 582-3)

Il s'agit d'une gestion qui, plutôt que de s'imposer, tend à se soustraire, à disparaître, à rendre la communication sociale aussi libre que possible.

Malgré cela, Péguy continuera probablement à être considéré comme ce solitaire marginal qui a donné sa vie pour une entreprise non viable. Son aventure s'est terminée trop tôt et on ne sait pas où elle aurait pu mener. Mais son œuvre subsiste toujours et fournit tous les éléments pour la juger de la manière la plus appropriée et pour en tirer de précieuses leçons théoriques dont il avait lui-même commencé à tirer les premiers éléments pour donner lieu à une théorie économique qui en est restée à son geste initial.

\section{Conclusion}

Le jeune Péguy est attiré par l'œuvre de Walras non pas par un intérêt momentané, mais par son approche bergsonienne : si Bergson enquête sur le temps intérieur qui échappe à la mécanique mathématique, Péguy enquête sur le temps historique qui échappe à l'économie mathématique. Pour ce dernier, la solution à la question sociale ne peut pas venir d'une théorie qui se prive de la possibilité de prendre en compte les contraintes réciproques entre les agents économiques, mais d'une investigation approfondie et directe de ces contraintes.

L'équilibre économique ne se trouve pas dans le système d'équations qui régule une mécanique de forces, mais entre les pressions qui peuvent être équilibrées par l'absorption dans le mouvement social. Péguy propose un retournement de méthode: commencer par la communication sociale hétérogène et à partir de là étudier la transmission des servitudes au lieu d'émettre des hypothèses sur des individus 
homogènes et libres de toute contrainte réciproque. Ainsi, Péguy impose le problème économique $d^{\prime}$ 'une manière radicalement différente de Walras et de ceux qui voient dans une organisation collectiviste la solution future et idéale à la question sociale. Pour Péguy, la solution ne se trouve pas dans une merveilleuse constitution, ni dans une propriété collective plutôt que privée.

La solution de Péguy est plus économique que juridique. Elle passe par l'organisation patiente des institutions économiques. Mais il ne leur suffit pas d'adopter une forme juridique collectiviste. Le dépassement de la subordination typique de la forme capitaliste ne peut être obtenu par le partage de l'autorité de commandement, mais par sa réduction à zéro. Ce qui compte n'est pas qui gère, mais quelle gestion est mise en place. Ce n'est pas le partage de la gestion qui compte pour l'action socialiste, mais une gestion ouverte aux propositions plurielles qui arrivent spontanément.

La Première Guerre mondiale n'a pas permis à Péguy de poursuivre ses recherches pratiques avec ses Cahiers, prototype d'une entreprise véritablement socialiste, et donc les réflexions économiques qu'il en tirait. Par contraste, les hypothèses de Walras concernant l'équilibre dans la production et la distribution de la richesse sociale étaient appelées à jouer un rôle de plus en plus central dans la formalisation de la science économique contemporaine. À l'écoute de Péguy, en revanche, le problème économique central sur lequel il faut se concentrer en vue d'un socialisme authentique et d'une authentique libération est plutôt de savoir comment la perte de liberté se transmet et comment elle peut être répartie et absorbée par une gestion qui recule et tend à disparaître.

Péguy a accordé toute l'attention requise au travail de Walras bien avant que beaucoup de gens ne se rendent compte de son importance. Mais il semble que la même chose se soit produite dans la direction opposée : avant tout le monde, Walras a réalisé l'importance des critiques et des réflexions économiques de Péguy. Mais, si Walras a pu travailler longtemps pour perfectionner sa théorie, la guerre a empêché Péguy d'achever sa pensée si profondément économique, comme en témoigne en particulier ses conférences de 1904.

Déjà à partir de 1905, la guerre qui se profile à I'horizon semble en partie occuper l'esprit de Péguy (Rioux, 2014), en lui laissant peut-être moins de temps pour ses réflexions économiques basées sur la distinction entre autorité de compétence et autorité de commandement. En 
effet, dans Notre patrie (1905), Péguy montre qu'il a bien conscience que la crise internationale de Tanger semble annoncer une invasion allemande à plus ou moins brève échéance. Toutefois, Péguy en parle comme d'une révélation seulement à la fin du cahier, qui est dédié à un autre événement : juste avant l'explosion de la crise de Tanger, le roi $d^{\prime}$ Espagne est en visite officielle à Paris. Comme le raconte Péguy dans ce cahier, tout le monde sort dans la rue pour voir son cortège. Péguy sort aussi, mais pour voir le peuple réuni ; un peuple attiré par la curiosité de voir le défilé d'un roi, mais en même temps le peuple révolutionnaire qui a défendu - et qui est toujours prêt à défendre - les valeurs républicaines. Voilà, incarnée dans ces valeurs, la patrie idéale de Péguy et de ceux qui partagent sa vision. Notre patrie parle en premier lieu de cela. Et c'est au nom de cette patrie mystique que Péguy voit en définitive un danger dans la politique pangermaniste. C'est au nom de cette même mystique républicaine, fondée sur l'autorité de conscience, qu'il a conduit son combat dans l'affaire Dreyfus, comme il I'expliquera dans Notre jeunesse (1910). C'est au nom de cette mystique, pour la liberté économique et politique, qu'il mettra toute son énergie dans l'aventure des Cahiers et qu'il s'engagera en 1914 contre le militarisme et l'impérialisme. Cette dernière résolution lui empêchera de poursuivre son œuvre. Mais, la Note sur M. Bergson et la philosophie bergsonienne (1914) ainsi que le texte posthume Note conjointe sur M. Descartes et la philosophie cartésienne (1914) laissent néanmoins présager de futures réflexions menées en profonde cohérence avec ses tous premiers engagements.

\section{Références}

Amato Massimo, 2009, «L'economia di un lavoro. Il pensiero dell'economico in Charles Péguy», dans Un poète l'a dit. Péguy di fronte alla contemporaneità, édité par Marisa Forcina et Michele Bee, Lecce, Milella, pp. 49-98.

Baranzini Roberto et Fèvre Raphaël, 2019, "Walras as an ordoliberal? », The European Journal of the History of Economic Thought, vol. 26, $\mathrm{n}^{\circ} 2$, pp. 380-413.

Bédarida François, 2002, "Histoire et mémoire chez Péguy », Vingtième Siècle. Revue d'histoire, vol. 73, n 1, pp. 101-110.

Bee Michele, 2009, "Leggere Péguy. Miseria, povertà e libertà di stile ", dans Un poète l'a dit. Péguy di fronte alla contemporaneità, édité par Marisa Forcina et Michele Bee, Milella, Lecce, pp. 125-146. 
Bee Michele, 2011, «Les correspondances entre économie et philosophie dans la pensée de Léon Walras et Charles Péguy », dans Léon Walras et l'équilibre économique général, Paris, Economica, pp. 79-98.

Bergson Henri, 1899, Essai sur les données immédiates de la conscience, Paris, PUF, 1958.

Bergson Henri, 1896, Matière et Mémoire, Paris, PUF, 2012.

Bridel Pascal, 1996, Le chêne et l'architecte. Un siècle de comptes rendus bibliographiques des 'Eléments d'économie politique pure' de Léon Walras : textes et commentaires, Genève, Droz.

Bridel Pascal et Huck Elisabeth, 2010, « Yet another look at Léon Walras's theory of tâtonnement », The European Journal of the History of Economic Thought, vol. 9, $\mathrm{n}^{\circ} 4$, pp. 513-540.

Creyghton Camille, 2011, «'Histoire, mémoire de l'humanité'. L'influence de Bergson sur la conception de l'histoire et celle de la mémoire de Charles Péguy ", L'Atelier du Centre de recherches historiques, $\mathrm{n}^{\circ} 7$.

Delaporte Jean, 1967, « Péguy et le socialisme scientifique (correspondance avec l'économiste Walras) », Esprit, vol. 356, n² 1, pp. 93-99.

Devaux André, 2000, «La rencontre Péguy-Bergson, ou le conflit entre une philosophie pure et une philosophie militante ", L'amitié Charles Péguy, $\mathrm{n}^{\circ}$ 92, pp. 429-436.

Dockès Pierre, 1990, «Les recensions de la première édition des Études d'économie sociale ", dans Léon Walras, Études d'économie sociale, Paris, Economica, pp. 483-507.

Dockès Pierre, 1996, La société n'est pas un pique-nique. Léon Walras et l'économie sociale, Paris, Economica.

Fantacci Luca, 2009, "Chi non gioca, perde. L'irrigidimento del denaro in Péguy e in Keynes ", dans Un poète l'a dit. Péguy di fronte alla contemporaneità, édité par Marisa Forcina et Michele Bee, Lecce, Milella, pp. 99-124.

Fédier François, 2014, «Péguy philosophe », dans Charles Péguy, édité par Camille Riquier, Paris, Les éditions du Cerf.

Gerbod Françoise, 2002, "Péguy, philosophe de l'histoire », Mil neuf cent, $\mathrm{n}^{\circ} 20$, pp. 12-13.

Guerlac Suzanne, 2017, Thinking in time. An introduction to Henri Bergson. Ithaca and London, Cornell University Press.

Halévy Daniel, 1918, Charles Péguy et les Cahiers de la Quinzaine, Paris, Payot.

Halévy Daniel, 1941, Charles Péguy et les Cahiers de la Quinzaine (édition augmentée), Préface de Robert Debré, Introduction et notes d'Éric Cahm, Paris, Grasset, 1979.

Jaffé William (éd.), 1965, Correspondence of Léon Walras and Related Papers, Amsterdam, North Holland. 
Latour Bruno, 2014, "Pourquoi Péguy se répète-t-il ? Péguy est-il illisible? », dans Charles Péguy, édité par Camille Riquier, Paris, Les éditions du Cerf, pp. 339-364.

Leroy Géraldi, 1973, «Péguy et l'économie mathématique », Revue $d^{\prime}$ Histoire littéraire de la France, $\mathrm{n}^{\circ} 2 / 3$, pp. 395-406.

Leroy Géraldi, 1975, "L'institution des Cahiers de la Quinzaine », dans Rencontres avec Péguy. Autour d'un Centenaire (1873-1973), Paris, Desclée De Brouwer, pp. 17-30.

Leroy Géraldi, 2014, Charles Péguy, Paris, Armand Colin.

Martin Auguste, 1968, "Le dossier Bergson-Péguy», Les études bergsoniennes, tome VIII, Paris, PUF, pp. 3-60.

Péguy Charles, 1897a, «Un économiste socialiste, M. Léon Walras », dans Cuvres en prose complètes, vol. 1, édité par Robert Burac, Paris, Gallimard, 1987, pp. 3-18.

Péguy Charles, 1897b, De la cité socialiste, dans CEuvres en prose complètes, vol. 1, édité par Robert Burac, Paris, Gallimard, 1987, pp. 34-39.

Péguy Charles, 1900, "Deuxième série au provincial », dans E Euvres en prose complètes, vol. 1, édité par Robert Burac, Paris, Gallimard, 1987, pp. 579-587.

Péguy Charles, 1901a, "Pour moi », dans Péguy et le Cahiers, textes choisis par Madame Charles Péguy, Paris, Gallimard, 1947, pp. 97-136.

Péguy Charles, 1901b, «Librairie des Cahiers », dans Euvres en prose complètes, vol. 1, édité par Robert Burac, Paris, Gallimard, 1987, pp. 1652-1656.

Péguy Charles, 1902a, "Personnalités », dans Péguy et le Cahiers, textes choisis par Madame Charles Péguy, Paris, Gallimard, 1947, pp.137-191.

Péguy Charles, 1902b, De Jean Coste, dans Euvres en prose complètes, vol. 1, édité par Robert Burac, Paris, Gallimard, 1987, pp. 1011-1057.

Péguy Charles, 1903 (posthume), «Emprunt des cahiers. De l'entraînement », dans Euvres en prose complètes, vol. 1, édité par Robert Burac, Paris, Gallimard, 1987, pp. 1271-1282.

Péguy Charles,1904a, "Cahiers de la Quinzaine V, 7 », dans Péguy et le Cahiers, textes choisis par Madame Charles Péguy, Paris, Gallimard, 1947, pp. 207-227.

Péguy Charles, 1904b (posthume), Leçons faites à l'École des Hautes Études Sociales, dans Euvres en prose complètes, vol. 1, édité par Robert Burac, Paris, Gallimard, 1987, pp. 1794-1827.

Péguy Charles, 1904c, "Avertissement », dans Euvres en prose complètes, vol. 1, édité par Robert Burac, Paris, Gallimard, 1987, pp. 1283-1317.

Péguy Charles, 1905, «Abonnement sur whatman» dans Péguy et le Cahiers, textes choisis par Madame Charles Péguy, Paris, Gallimard, 1947 , pp. 258-268. 
Péguy Charles, 1910, Notre jeunesse, dans Euvres en prose complètes, vol. 3, édité par Robert Burac, Paris, Gallimard, 1992, pp. 5-159.

Péguy Charles, 1912-3 (posthume), Clio, dialogue de l'histoire et de l'âme païenne, dans Euvres en prose complètes, vol. 3, édité par Robert Burac, Paris, Gallimard, 1992, pp. 997-1214.

Péguy Charles, 1913, L'argent suite, dans Euvres en prose complètes, vol. 3, édité par Robert Burac, Paris, Gallimard, 1992, pp. 848-996.

Péguy Charles, 1914, Note sur M. Bergson et la philosophie bergsonienne, dans CEuvres en prose complètes, vol. 3, édité par Robert Burac, Paris, Gallimard, 1992, pp. 1246-1277.

Péguy Charles, 1914 (posthume), Note conjointe sur M. Descartes et la philosophie cartésienne, dans Euvres en prose complètes, vol. 3, édité par Robert Burac, Paris, Gallimard, 1992, pp. 1278-1478.

Potier Jean-Pierre, 1994, "Classification des sciences et divisions de l'Économie politique et sociale' dans l'œuvre de Léon Walras : une tentative de reconstruction ", Économies et Sociétés, vol. 28, n 10-11, pp. 223-277.

Riquier Camille, 2009, Archéologie de Bergson. Temps et métaphysique. Paris, PUF.

Riquier Camille, 2014, « Péguy 'Bergsonien'. La mémoire, l'histoire, l'inoubliable", dans Charles Péguy, édité par Camille Riquier, Paris, Les éditions du Cerf, pp. 149-178.

Riquier Camille, 2017, Philosophie de Péguy ou les mémoires d'un imbécile, Paris, PUF.

Rioux Jean-Pierre, 2014, La mort du lieutenant Péguy, Paris, Tallandier.

Robinet André, 1968, Péguy entre Jaurès, Bergson et l'Église. Métaphysique et politique, Paris, Seghers.

Rolland Romain, 1946, Péguy, Buenos Aires, Viau-Feugere.

Saatdjian Dominique, 2001, « Péguy : la réalité à répétition », in Hommage à François Fédier, édité par Hadrien France-Lanord et Fabrice Midal, Paris, Lettrage, pp. 349-363.

Saatdjian Dominique, 2011, "Préface», dans Charles Péguy: une éthique sans compromis, Paris, Pocket, pp. 11-17.

Smith Robert J., 1973, «L'atmosphère politique à l'École Normale Supérieure à la fin du XIX ${ }^{\mathrm{e}}$ siècle ", Revue d'histoire moderne et contemporaine, vol. 20, $\mathrm{n}^{\circ} 2$, pp. 248-268.

Sorel Georges, 1904, «Lettre à Péguy du 20 janvier 1904 », dans Euvres en prose complètes, vol. 1, éditées par Robert Burac, Paris, Gallimard, 1987, p. 1786.

Thibaud Paul, 1975, «La pensée économique de Péguy», dans Rencontres avec Péguy. Autour d'un Centenaire (1873-1973), Paris, Desclée De Brouwer, pp. 137-147. 
Thibaud Paul, 1978, «La liberté et la communication ». Esprit, vol. 22, $\mathrm{n}^{\circ} 10, \mathrm{pp} .36-42$.

Viard Jacques, 1969, «Les œuvres posthumes de Charles Péguy », Cahiers de l'Amitié Charles Péguy, 23, Paris, Minard.

Vitry Alexandre de, 2015, Conspirations d'un solitaire. L'individualisme civique de Charles Péguy, Paris, Les Belles Lettres.

Walras Léon, 1874, Éléments d'économie politique pure, dans Auguste et Léon Walras œuvres économiques complètes, vol. 8, édité par Claude Mouchot, Paris, Economica, 1988.

Walras Léon, 1896, Études d'économie sociale, dans Auguste et Léon Walras ouvres économiques complètes, vol. 9, édité par Pierre Dockès, Paris, Economica, 1990.

Worms Frédéric, 1994, «Les trois dimensions de la question de l'espace chez Bergson », Epokhé, n 4, pp. 89-116.

Worms Frédéric, 2000, «Péguy auditeur de Bergson : entre deux écritures », L'amitié Charles Péguy, n 92, pp. 461-466.

Worms Frédéric, 2011, «Lire Bergson: un apprentissage», dans Lire Bergson, édité par Frédéric Worms et Camille Riquier, Paris, PUF, pp. 3-20. 
ANNEXE. Correspondance entre Charles Péguy et Léon Walras : extraits

\section{Document 1 : Lettre de Péguy à Walras du 19 février 1897 (recto)}

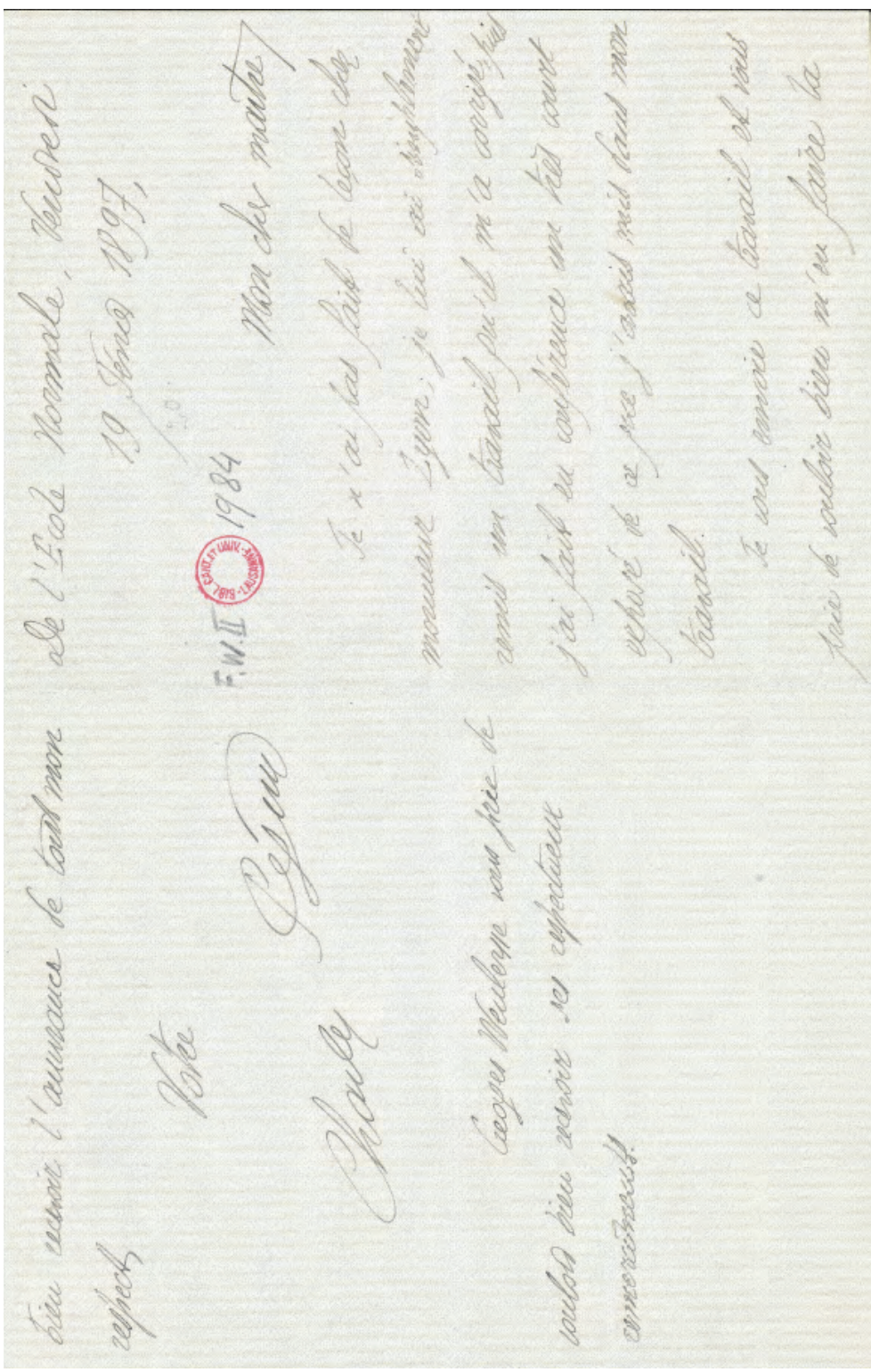

Source: Fonds Walras de la Bibliothèque de I'Université de Lausanne. 
Document 1 : Lettre de Péguy à Walras du 19 février 1897 (verso)

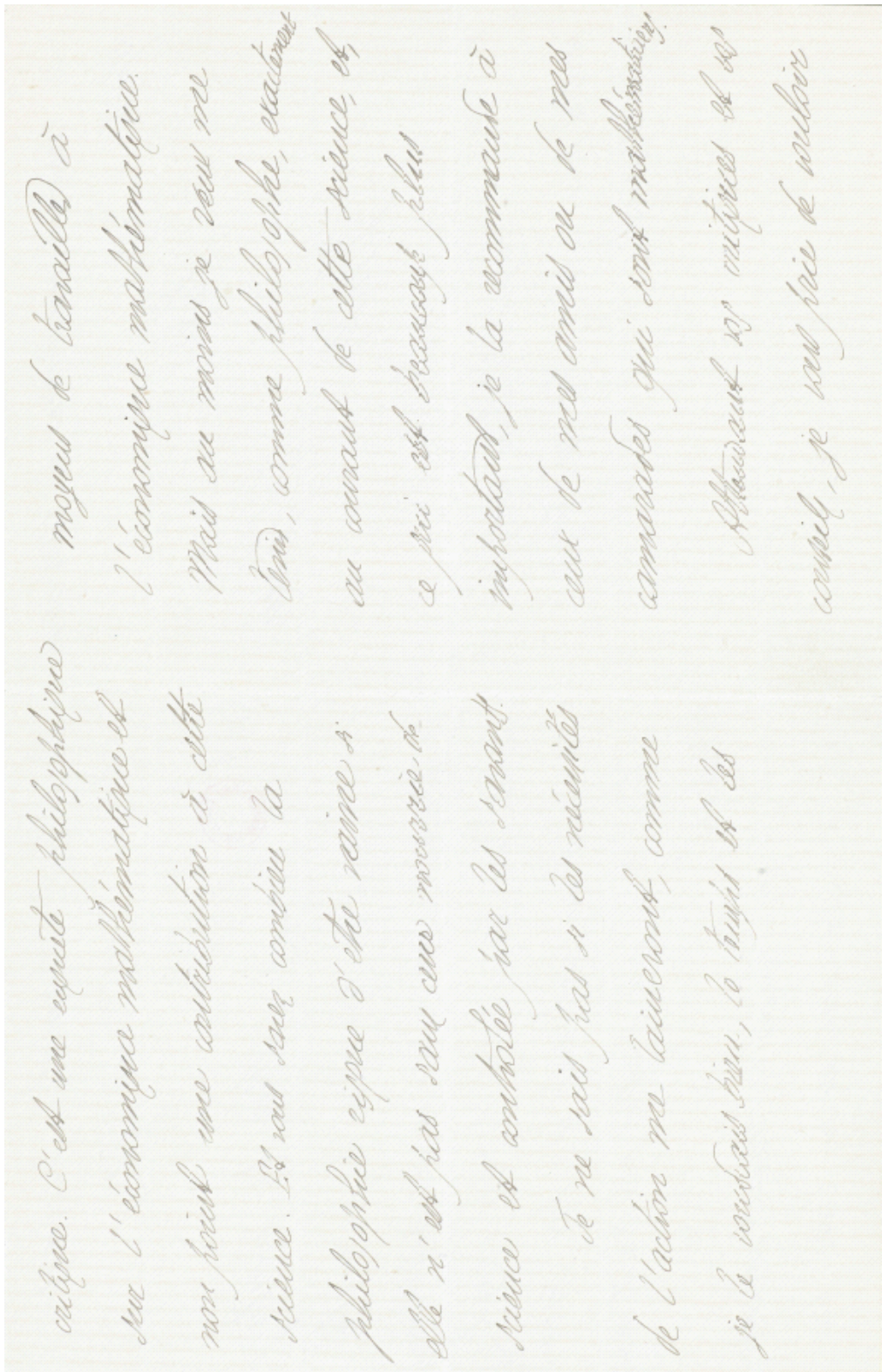

Source: Fonds Walras de la Bibliothèque de I'Université de Lausanne. 
Document 2. Brouillon de la lettre de Walras à Péguy du 31 mars 1897

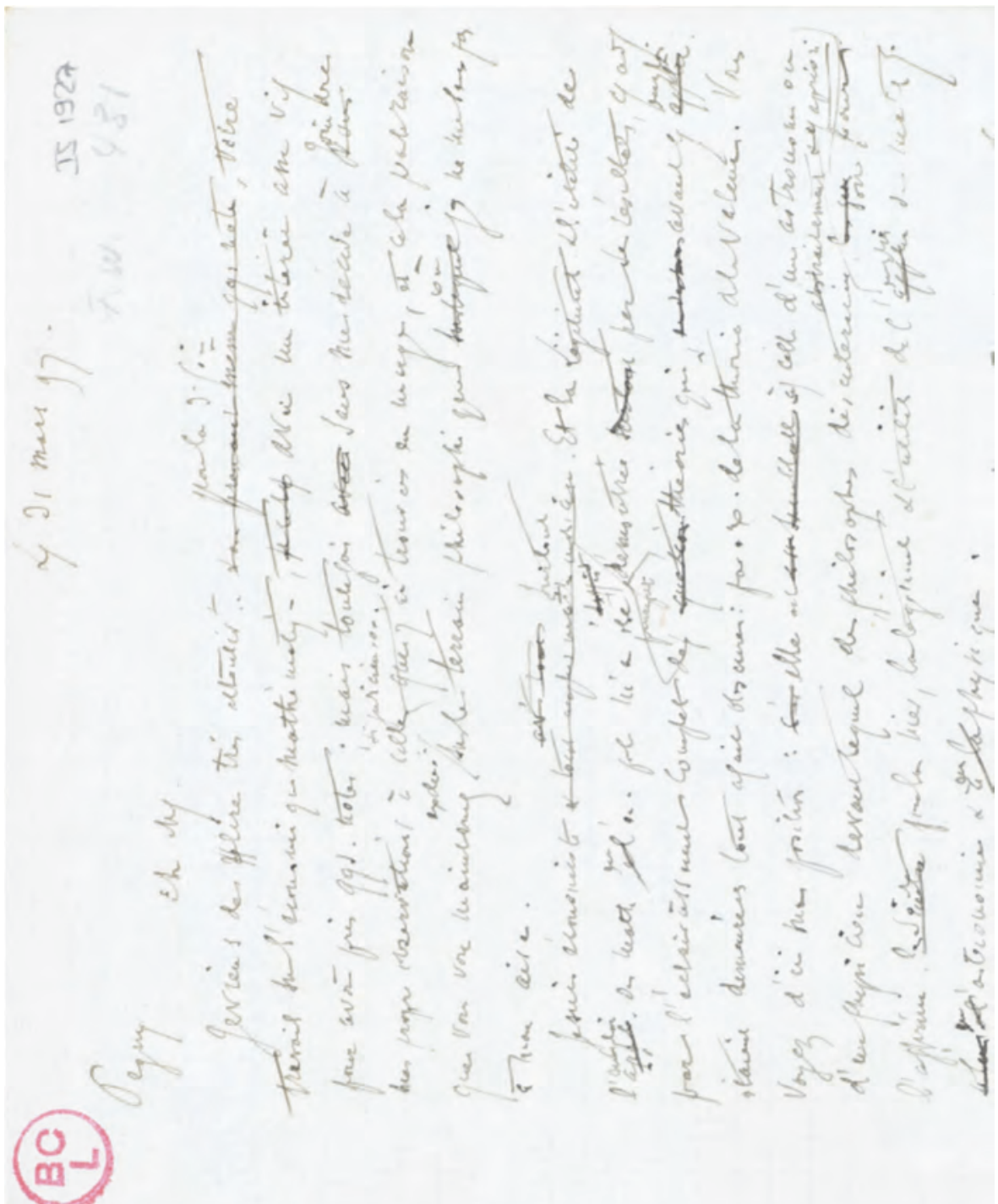

Source : Fonds Walras de la Bibliothèque de I'Université de Lausanne. 
Document 2 (suite et fin) : Brouillon de la lettre de Walras à Péguy du 31 mars 1897

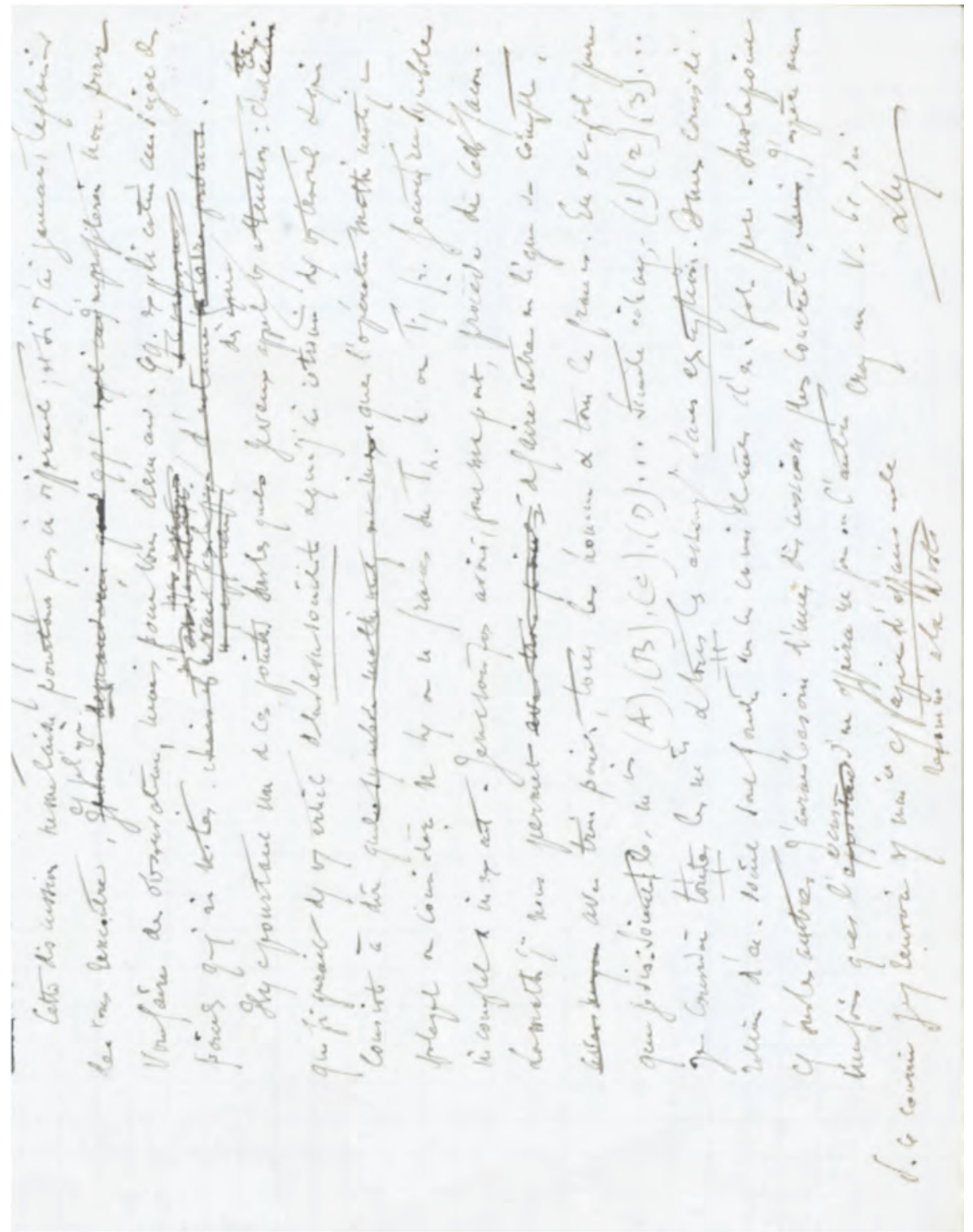

Source : Fonds Walras de la Bibliothèque de I'Université de Lausanne. 
